\title{
The Asymptotic Behavior in a Nonlinear Cobweb Model with Time Delays
}

\author{
Akio Matsumoto ${ }^{1}$ and Ferenc Szidarovszky ${ }^{2}$ \\ ${ }^{1}$ Department of Economics, International Center for Further Development of Dynamic Economic Research, Chuo University, \\ 742-1 Higashi-Nakano, Hachioji, Tokyo 192-0393, Japan \\ ${ }^{2}$ Department of Applied Mathematics, University of Pécs, Ifjúság Útja 6, Pécs 7624, Hungary
}

Correspondence should be addressed to Ferenc Szidarovszky; szidarka@gmail.com

Received 24 March 2015; Accepted 29 June 2015

Academic Editor: Peng Shi

Copyright (C) 2015 A. Matsumoto and F. Szidarovszky. This is an open access article distributed under the Creative Commons Attribution License, which permits unrestricted use, distribution, and reproduction in any medium, provided the original work is properly cited.

We study the effects of production delays on the local as well as global dynamics of nonlinear cobweb models in a continuous-time framework. After reviewing a single delay model, we proceed to two models with two delays. When the two delays are used to form an expected price or feedback for price adjustment, we have a winding stability switching curve and in consequence obtain repetition of stability losses and gains via Hopf bifurcation. When the two delays are involved in two interrelated markets, we find that the stability switching occurs on straight lines and complicated dynamics can arise in unstable markets.

\section{Introduction}

It is now well known that the cobweb model or cobweb theory has been developed in various directions since the pioneering work of Kaldor [1]. It explains why and how certain types of markets give rise to fluctuations in prices and quantities. Since it mainly focuses on the agricultural markets in which producers determine their outputs before observing market prices and a delay between planting and harvesting is inevitable, its key issues are an expectation formation of price and a production delay. In early stage, the models are essentially linear and constructed in discrete-time scales in which production delay is incorporated from the beginning. Thus the main question is on how the expectation formations such as naive, adaptive, and rational expectations are responsible for the emergence of fluctuations. During the last two decades, increasing attention has been given to nonlinear dynamics. A summary of recent developments in nonlinear oligopolies is given in Bischi et al. [2] with a wide variety of the extensions of the classical Cournot model. More recently, Shirai and Amano [3] examine a production process with nonlinearity of the rate of return on sales. Their mathematical model is based on a Van der Pol differential equation. Nonlinear models such as discrete-time cobweb models can generate a wide spectrum of dynamic behaviors involving chaos. See Dieci and Westerhoff [4] and Hommes [5] as additional references, to name a few.

It is, however, less known that a continuous-time cobweb model with fixed time delay is also developed with the same problem consciousness as early as in the 1930s. In particular, Haldane [6] found the similarity between the effects caused by the rise in the birth rate in biology and the ones caused by a rise in commodity price in economics and built a simple economic model to examine the fluctuations in price and the rate of production, coaxing the idea from theoretical biology. Independently from Haldane, Larson [7] presents a linear continuous-time model in which a hog cycle is described as a harmonic motion. It is assumed that realized production has 12 -month delay from planned production and the rate of production change is proportional to the deviation of price from equilibrium. Mackey [8] gives a nonlinear price adjustment model with production delay and rigorously derives a stability switching condition for which the stability of equilibrium is lost. Furthermore, it is shown that a Hopf bifurcation takes place and thus the stable equilibrium bifurcates to a limit cycle after the loss of stability. Recently Gori et al. [9] propose a delay cobweb model with the profit-maximizing behavior to characterize production 
cycles. Although the delay models have been an object of study for a long time, these are subject to a single delay and little is known about multiple delay models.

The theory of delay differential equations is well known from the mathematical literature. The classical book of Bellman and Cooke [10] summarizes the earlier results. A simple analytic method of examining systems with a single delay is given, for example, in Matsumoto and Szidarovszky [11], and it is extended to a special class of models with two delays in Matsumoto and Szidarovszky [12]. A more general geometric approach is introduced in Gu et al. [13], which can be used to find the stability switching curves and the directions of the stability switches on these curves. Their method is further improved by Lin and Wang [14] giving the tool for examining more complex systems.

The purpose of this study is, based on Mackey's formulation, to investigate how multiple delays affect cobweb price dynamics, applying the recent mathematical developments to characterize the stability of two-delay differential equations developed by Gu et al. [13] and Lin and Wang [14]. Two main results demonstrated in this paper are the following:

(i) Simple dynamics emerge but stability losses and gains are repeatedly taking place in a single market with two time delays.

(ii) No stability gain occurs but complex dynamics can arise when two markets with two delays are unstable.

This paper is organized as follows. In Section 2, a continuous-time nonlinear price adjustment model is presented as a basic model. In Section 3, a single production delay is introduced to review how the delay affects dynamics. In Section 4, the model with two production delays is constructed and the stability switching curve is analytically and numerically derived. In Section 5, two markets' models with two delays are considered to develop the conditions under which the two markets are stable or unstable. It is shown that various dynamics arise when the two markets are unstable. In the final section, concluding remarks and further research directions are given.

\section{Basic Cobweb Model}

As in Mackey [8], we consider price dynamics in a continuous-time framework in which relative variations in market price $p(t)$ are adjusted to be proportional to excess demand:

$$
\frac{\dot{p}(t)}{p(t)}=K\left[D(p(t))-S\left(p^{e}(t)\right)\right],
$$

where $K>0$ is the adjustment coefficient, $p^{e}$ is the expected price, and $D(p)$ and $S\left(p^{e}\right)$ are the demand and supply functions of commodity to be considered. Following the tradition, it is assumed that demand negatively depends on price while supply positively depends on the expected price. For the sake of analytical simplicity it is also assumed that consumers and producers make their decisions based only on the price information appearing in the good market.
This assumption is taken away in Section 5. The expected price is formed based on the past observed prices:

$$
p^{e}(t)=F\left[p\left(t-\tau_{1}\right), p\left(t-\tau_{2}\right), \ldots, p\left(t-\tau_{n}\right)\right],
$$

where $\tau_{i}>0$ for $i=1,2, \ldots, n$ and $p\left(t-\tau_{i}\right)$ is the delayed price or the price realized at time $t-\tau_{i}$. Again for the sake of simplicity, demand and supply functions are assumed to be linear:

$$
\begin{gathered}
D(t)=d_{1}-d_{2} p(t) \quad \text { with } d_{1}>0, d_{2}>0, \\
S(t)=s_{1}+s_{2} p^{e}(t) \quad \text { with } s_{1}>0, s_{2}>0 .
\end{gathered}
$$

The equilibrium price and quantity satisfy the conditions of $p^{*}=p^{e}(t)=p(t)$ and $q^{*}=D\left(p^{*}\right)=S\left(p^{*}\right)$ and are obtained as

$$
\begin{aligned}
& p^{*}=\frac{d_{1}-s_{1}}{d_{2}+s_{2}}, \\
& q^{*}=\frac{d_{1} s_{2}+d_{2} s_{1}}{d_{2}+s_{2}},
\end{aligned}
$$

where, for positivity of the equilibrium price, $d_{1}>s_{1}$ is assumed. This is a natural assumption requiring that the maximum demand exceeds the minimum supply.

Substituting (3) and (4) into (1), taking $p^{e}(t)=p(t)$, and then multiplying both sides of the resultant equation by $p(t)$ yield a nonlinear price adjustment equation:

$$
\dot{p}(t)=K p(t)\left[d_{1}-s_{1}-\left(d_{2}+s_{2}\right) p(t)\right] .
$$

The equilibrium price $p^{*}$ is also a stationary point. ${ }^{1}$ To examine stability of the equilibrium price, we denote the right-hand side of (6) by $G_{1}(p(t))$ and linearize it around $p=p^{*}$ :

$$
\dot{p}_{\delta}(t)=\left.\frac{d G_{1}(p(t))}{d p(t)}\right|_{p=p^{*}} p_{\delta}(t)
$$

or

$$
\dot{p}_{\delta}(t)=-k\left(d_{2}+s_{2}\right) p_{\delta}(t),
$$

where $p_{\delta}(t)=p(t)-p^{*}$ and $k=K p^{*}$. Its solution is

$$
p(t)=p^{*}+\left(p(0)-p^{*}\right) e^{-k\left(d_{2}+s_{2}\right) t} .
$$

Since $k\left(d_{2}+s_{2}\right)>0$, the equilibrium price is always locally stable meaning that the market price converges to the equilibrium price as $t \rightarrow \infty$ if the initial price is close enough to the equilibrium.

\section{Cobweb Model with a Single Delay}

A production time delay is introduced into basic model (6). Concerning the expectation formation, we start with the simplest form of $F(\cdot)$ where the expected price at time $t$ is the market price realized at time $t-\tau$ with $\tau>0$. 
Assumption 1. Consider $p^{e}(t)=p(t-\tau)>0$.

Accordingly, the supply function is modified as

$$
S(t)=s_{1}+s_{2} p(t-\tau)
$$

Substituting (10) into (6) presents a delay price adjustment equation:

$$
\dot{p}(t)=K p(t)\left[d_{1}-s_{1}-d_{2} p(t)-s_{2} p(t-\tau)\right]
$$

which is a first-order nonlinear delay differential equation. It can be confirmed that $p^{*}$ is also a unique positive stationary state of (11). If $G_{2}(p(t), p(t-\tau))$ denotes the right-hand side of (11), then a linearized equation in a neighborhood of the stationary point $\mathbf{p}_{2}^{*}=\left(p^{*}, p^{*}\right)$ is

$$
\dot{p}_{\delta}(t)=\left.\frac{\partial G_{2}}{\partial p(t)}\right|_{\mathbf{p}_{2}^{*}} p_{\delta}(t)+\left.\frac{\partial G_{2}}{\partial p(t-\tau)}\right|_{\mathbf{p}_{2}^{*}} p_{\delta}(t-\tau)
$$

or

$$
\dot{p}_{\delta}(t)=-k d_{2} p_{\delta}(t)-k s_{2} p_{\delta}(t-\tau)
$$

Introducing the new variable $x(t)=p_{\delta}(t)$ and the new parameters, $\alpha=k d_{2}$ and $\beta=k s_{2}$, we obtain the following form of the linearized equation:

$$
\dot{x}(t)+\alpha x(t)+\beta x(t-\tau)=0,
$$

where $x^{*}=x(t)=0$ is the only stationary point. Assuming an exponential solution

$$
x(t)=e^{\lambda t} u
$$

and substituting it into (14) give the corresponding characteristic equation

$$
\lambda+\alpha+\beta e^{-\lambda \tau}=0 .
$$

Without delay $\tau=0$, the stationary point is locally asymptotically stable. If stability of the trivial solution $x(t)=$ 0 of (14) switches to instability at $\tau=\bar{\tau}$, then (16) must have a pair of pure conjugate imaginary roots. It is then assumed, without loss of generality, that $\lambda=i \omega$ with $\omega>0$ is a root. Substituting it into (16) breaks down the characteristic equation to the real and imaginary parts:

$$
\begin{aligned}
& \alpha+\beta \cos \tau \omega=0, \\
& \omega-\beta \sin \tau \omega=0 .
\end{aligned}
$$

Moving the constant terms to the right-hand sides and adding the squares of the resulting equations give

$$
\omega^{2}=k^{2}\left(s_{2}+d_{2}\right)\left(s_{2}-d_{2}\right) .
$$

If $s_{2} \leq d_{2}$, then there is no $\omega>0$, implying that the delay is harmless.

Theorem 2. If $s_{2} \leq d_{2}$, then the positive steady state of (6) is locally asymptotically stable for any positive values of $\tau$.
On the other hand, if $s_{2}>d_{2}$, then we can define $\bar{\omega}>0$ as

$$
\bar{\omega}=k \sqrt{\left(s_{2}+d_{2}\right)\left(s_{2}-d_{2}\right)} .
$$

It is substituted into (17) to obtain threshold values of $\tau:^{3}$

$$
\bar{\tau}=\frac{1}{\bar{\omega}}\left[\cos ^{-1}\left(-\frac{d_{2}}{s_{2}}\right)+2 n \pi\right] \quad(n=0,1,2, \ldots) .
$$

In order to determine the direction of the stability switch, we can think of the root of (16) as a continuous function of the delay $\tau$. Then differentiating (16) with respect to $\tau$ and arranging terms yield

$$
\left(\frac{d \lambda}{d \tau}\right)^{-1}=\frac{1-\tau \beta e^{-\lambda \tau}}{\lambda \beta e^{-\lambda \tau}}
$$

Thus

$$
\begin{aligned}
\left(\frac{d(\operatorname{Re} \lambda)}{d \tau}\right)_{\lambda=i \omega}^{-1} & =\operatorname{Re}\left[\frac{1-\tau \beta e^{-\lambda \tau}}{\lambda \beta e^{-\lambda \tau}}\right]_{\lambda=i \omega} \\
& =\operatorname{Re}\left[-\frac{1}{\lambda(\lambda+\alpha)}\right]_{\lambda=i \omega} \\
& =\operatorname{Re}\left[\frac{\omega+i \alpha}{\omega\left(\omega^{2}+\alpha^{2}\right)}\right]=\frac{1}{\omega^{2}+\alpha^{2}} .
\end{aligned}
$$

Hence

$$
\left.\frac{d(\operatorname{Re} \lambda)}{d \tau}\right|_{\lambda=i \omega}>0
$$

This inequality implies that all roots that cross the imaginary axis at $i \omega$ cross from left to right as $\tau$ increases. So at point $\bar{\tau}$ with $n=0$ stability is lost and cannot be regained later.

Further substituting $\bar{\omega}$ into (21) determines the critical value of the delay

$$
\bar{\tau}\left(d_{2}, s_{2}\right)=\frac{\cos ^{-1}\left(-d_{2} / s_{2}\right)}{K p^{*} \sqrt{\left(s_{2}+d_{2}\right)\left(s_{2}-d_{2}\right)}}
$$

with

$$
\begin{aligned}
& \frac{\partial \bar{\tau}}{\partial d_{2}} \\
& =\frac{s_{2} \sqrt{1-\left(d_{2} / s_{2}\right)^{2}}\left[\left(s_{2}^{2}-d_{2}^{2}\right)+s_{2}^{2} \sqrt{1-\left(d_{2} / s_{2}\right)^{2}} \cos ^{-1}\left(-d_{2} / s_{2}\right)\right]}{K\left(d_{1}-s_{1}\right)\left(s_{2}-d_{2}\right)\left(s_{2}^{2}-d_{2}^{2}\right)^{3 / 2}} \\
& >0, \\
& \frac{\partial \bar{\tau}}{\partial s_{2}} \\
& =-\frac{d_{2} \sqrt{1-\left(d_{2} / s_{2}\right)^{2}}\left[\left(s_{2}^{2}-d_{2}^{2}\right)+s_{2}^{2} \sqrt{1-\left(d_{2} / s_{2}\right)^{2}} \cos ^{-1}\left(-d_{2} / s_{2}\right)\right]}{K\left(d_{1}-s_{1}\right)\left(s_{2}-d_{2}\right)\left(s_{2}^{2}-d_{2}^{2}\right)^{3 / 2}} \\
& <0 .
\end{aligned}
$$

In Figure 1(a), the stability switching curve is depicted as a hyperbolic curve on which the real parts of the eigenvalues 


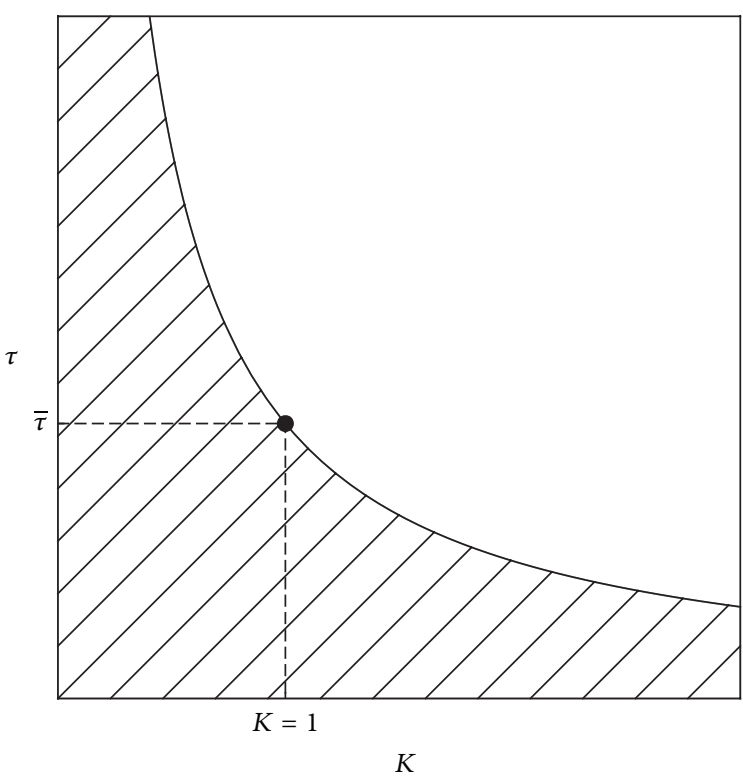

(a) Stability region

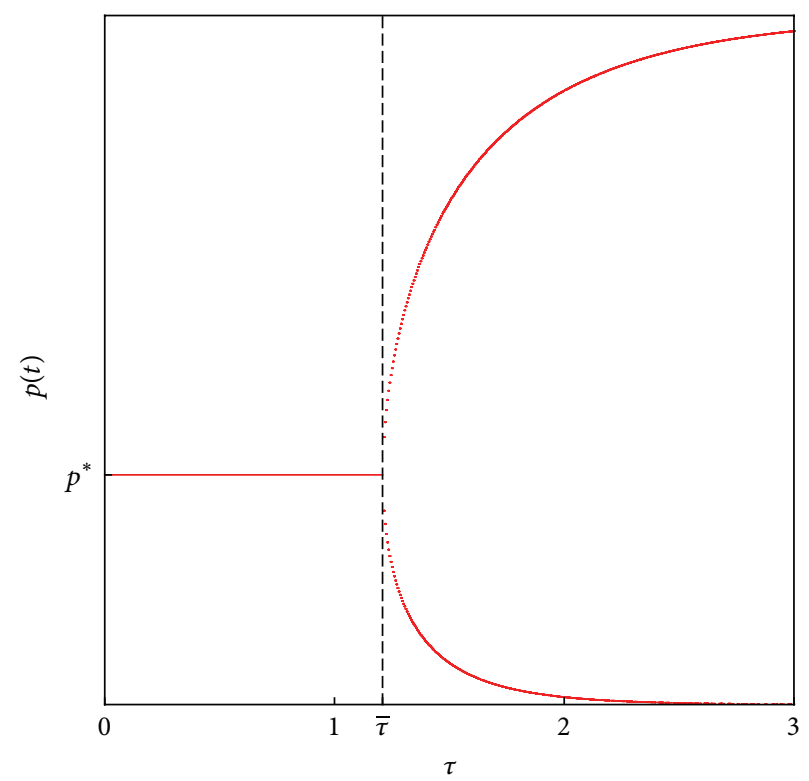

(b) Bifurcation diagram

FIGURE 1: Numerical results on one-delay model (11).

are zero. The equilibrium price is stable in the shaded region below the curve and unstable in the white region above. Since $\partial \bar{\tau} / \partial d_{2}>0$ and $\partial \bar{\tau} / \partial s_{2}<0$, increasing the value of $d_{2}$ and decreasing the value of $s_{2}$ shift the stability switching curve upward, implying that those parameter changes enlarge the stability region and thus have stabilizing effects. Figure 1(b) illustrates the bifurcation diagram with respect to $\tau$ where the parameters are specified as follows.

Assumption 3. Consider $K=1, d_{1}=4, s_{1}=1, d_{2}=1$, and $s_{2}=2$.

Notice that the maximum demand $\left(d_{1}\right)$ is larger than the minimal supply $\left(s_{1}\right)$, so the nonzero equilibrium is positive. Furthermore the condition of Theorem 2 is violated, so stability switch can be observed. The diagram is constructed in the following way. With a fixed value of $\tau$, we run delay system (11) for $0 \leq t \leq 1000$. To take away the initial disturbance, we discard the data of $p(t)$ for $t \leq 950$ and plot the local maximum and local minimum of $p(t)$ for $950 \leq t \leq$ 1000 against the value of $\tau$. The value of $\tau$ is increased with an increment 0.01 and then the same procedure is repeated until $\tau$ arrives at 3 . Under these circumstances, when $K=1$, the threshold value of $\tau$ denoted by $\bar{\tau}$ is obtained as

$$
\bar{\tau}\left(d_{2}, s_{2}\right)=\frac{2 \pi}{3 \sqrt{3}} \simeq 1.209 .
$$

It is seen in Figure 1(a) that the equilibrium is stable for $\tau<\bar{\tau}$ and unstable for $\tau>\bar{\tau}$. The two branches of the diagram given in Figure 1(b) indicate two issues; one is that a trajectory has one maximum and one minimum, implying the birth of a limit cycle which is confirmed by Hopf bifurcation theorem with (24), and the other is that a cycle becomes larger as the length of the delay increases. Theorem 4 summarizes the results.
Theorem 4. Given $s_{2}>d_{2}$, the positive steady state of (11) is locally asymptotically stable if $0<\tau<\bar{\tau}$, loses stability at $\tau=\bar{\tau}$, and bifurcates to a limit cycle if $\tau>\bar{\tau}$, where $\bar{\tau}=\bar{\tau}(1,2) \simeq$ 1.209 .

\section{Cobweb Model with Two Delays}

The price expectation is formed naively under Assumption 1 . Convergence to the stationary point occurs for $\tau<\bar{\tau}$. The producers with delayed price information can eventually arrive at the stationary state. On the other hand, since cyclic oscillation arises for $\tau>\bar{\tau}$, the producers sooner or later realize that their expectations are systematically wrong. As a natural consequence, even though they are assumed to be boundedly rational, the producers may change somehow the way they form expectations. One possible way is to utilize more price information obtained in the past. To make it simpler, the producers are assumed to use different prices at times, $t-\tau_{1}$ and $t-\tau_{2}$. There are at least two different ways to employ these two prices. One is to make the price expectation as the weighted average of these prices and the other is to use the difference between these two prices as delay feedback. We first make the following assumption which is a direct extension of Assumption 1 when the price expectation formation is the weighted average of the past two prices.

Assumption 5. Consider $p^{e}(t)=\theta p\left(t-\tau_{1}\right)+(1-\theta) p\left(t-\tau_{2}\right)$ with $0<\theta<1$.

Accordingly the supply function is modified as

$$
S\left(p^{e}(t)\right)=s_{1}+s_{2}\left[\theta p\left(t-\tau_{1}\right)+(1-\theta) p\left(t-\tau_{2}\right)\right]
$$


and then the price adjustment is governed by a two-delay differential equation:

$$
\begin{aligned}
& \dot{p}(t)=K p(t)\left[\left(d_{1}-s_{1}\right)-d_{2} p(t)-s_{2} \theta p\left(t-\tau_{1}\right)\right. \\
& \left.\quad-s_{2}(1-\theta) p\left(t-\tau_{2}\right)\right] .
\end{aligned}
$$

It is clear that the equilibrium price is a unique positive stationary point of (29). To examine local dynamics, we let $G_{3}\left(p(t), p\left(t-\tau_{1}\right), p\left(t-\tau_{2}\right)\right)$ be the right-hand side of (29). The linear approximation in the neighborhood of the stationary point $\mathbf{p}_{3}^{*}=\left(p^{*}, p^{*}, p^{*}\right)$ is

$$
\begin{aligned}
\dot{p}_{\delta}(t)= & \left.\frac{\partial G_{3}}{\partial p(t)}\right|_{\mathbf{p}_{3}^{*}} p_{\delta}(t)+\left.\frac{\partial G_{3}}{\partial p\left(t-\tau_{1}\right)}\right|_{\mathbf{p}_{3}^{*}} p_{\delta}\left(t-\tau_{1}\right) \\
& +\left.\frac{\partial G_{3}}{\partial p\left(t-\tau_{2}\right)}\right|_{\mathbf{p}_{3}^{*}} p_{\delta}\left(t-\tau_{2}\right)
\end{aligned}
$$

or

$$
\begin{aligned}
\dot{p}_{\delta}(t)= & -k d_{2} p_{\delta}(t)-k s_{2} \theta p_{\delta}\left(t-\tau_{1}\right) \\
& -k s_{2}(1-\theta) p_{\delta}\left(t-\tau_{2}\right) .
\end{aligned}
$$

As before, the last form can be reduced to

$$
\dot{x}(t)+\alpha x(t)+\beta x\left(t-\tau_{1}\right)+\gamma x\left(t-\tau_{2}\right)=0
$$

with

$$
\begin{aligned}
& \alpha=k d_{2}, \\
& \beta=k s_{2} \theta, \\
& \gamma=k s_{2}(1-\theta) .
\end{aligned}
$$

We now turn our attention to the delay feedback with which price adjustment equation (1) is modified as

$$
\begin{aligned}
\frac{\dot{p}(t)}{p(t)}= & k_{1}\left[\left(d_{1}-s_{1}\right)-d_{2} p(t)-s_{2} p\left(t-\tau_{1}\right)\right] \\
& +k_{2}\left[p\left(t-\tau_{1}\right)-p\left(t-\tau_{2}\right)\right]
\end{aligned}
$$

where $k_{1}$ is an adjustment coefficient and $k_{2}$ is a coefficient of the feedback. This can be rewritten as a differential equation with two delays:

$$
\begin{aligned}
\dot{p}(t) & =k_{1} p(t)\left[\left(d_{1}-s_{1}\right)-d_{2} p(t)\right. \\
& \left.-s_{2}\left(1-\frac{k_{2}}{s_{2} k_{1}}\right) p\left(t-\tau_{1}\right)-s_{2} \frac{k_{2}}{s_{2} k_{1}} p\left(t-\tau_{2}\right)\right] .
\end{aligned}
$$

If we assume $k_{2}<s_{2} k_{1}$ and denote

$$
\begin{aligned}
1-\frac{k_{2}}{s_{2} k_{1}} & =\theta, \\
\frac{k_{2}}{s_{2} k_{1}} & =1-\theta,
\end{aligned}
$$

then the differential equation with the delay feedback (35) is identical with (29), the differential equation with the average price. Hence these equations generate essentially the same dynamics, although their economic interpretations are definitely different. In the following we focus on (29) only because the number of the parameters is smaller; however, the same results can be obtained by examining (35).

The corresponding characteristic equation of (32) is obtained by substituting an exponential solution $x(t)=e^{\lambda t} u$ :

$$
\lambda+a+\beta e^{-\lambda \tau_{1}}+\gamma e^{-\lambda \tau_{2}}=0 .
$$

Stability of (32) depends on the locations of the eigenvalues of (37) that is investigated by applying the method developed by $\mathrm{Gu}$ et al. [13]. Dividing both sides of (37) by $\lambda+a$ and introducing the new functions

$$
\begin{aligned}
& a_{1}(\lambda)=\frac{\beta}{\lambda+a}, \\
& a_{2}(\lambda)=\frac{\gamma}{\lambda+\alpha}
\end{aligned}
$$

simplify the left-hand side of (37):

$$
a(\lambda)=1+a_{1}(\lambda) e^{-\lambda \tau_{1}}+a_{2}(\lambda) e^{-\lambda \tau_{2}} .
$$

Substituting a possible solution $\lambda=i \omega$ with $\omega>0$ into the two equations of (38) results in

$$
\begin{aligned}
& a_{1}(i \omega)=\frac{a \beta}{\alpha^{2}+\omega^{2}}-i \frac{\beta \omega}{\alpha^{2}+\omega^{2}}, \\
& a_{2}(i \omega)=\frac{a \gamma}{\alpha^{2}+\omega^{2}}-i \frac{\gamma \omega}{\alpha^{2}+\omega^{2}} .
\end{aligned}
$$

Their absolute values are

$$
\begin{aligned}
& \left|a_{1}(i \omega)\right|=\frac{\beta}{\sqrt{\alpha^{2}+\omega^{2}}}, \\
& \left|a_{2}(i \omega)\right|=\frac{\gamma}{\sqrt{\alpha^{2}+\omega^{2}}} .
\end{aligned}
$$

We can consider the three terms in (39) as three vectors in the complex plane with the magnitudes $1,\left|a_{1}(\lambda)\right|$, and $\left|a_{2}(\lambda)\right|$. The solution of $a(\lambda)=0$ means that these vectors form a triangle if we put them head to tail. That is, solving $a(\lambda)=0$ algebraically is equivalent to constructing a triangle geometrically with the following three conditions:

$$
\begin{aligned}
1 & \leq\left|a_{1}(i \omega)\right|+\left|a_{2}(i \omega)\right|, \\
\left|a_{1}(i \omega)\right| & \leq 1+\left|a_{2}(i \omega)\right|, \\
\left|a_{2}(i \omega)\right| & \leq 1+\left|a_{1}(i \omega)\right| .
\end{aligned}
$$

Substituting the absolute values of (41) and (42) converts these three conditions to the following conditions:

$$
(\beta-\gamma)^{2}-\alpha^{2} \leq \omega^{2} \leq(\beta+\gamma)^{2}-\alpha^{2}
$$

that can be rewritten as

$$
k^{2}\left[s_{2}^{2}(2 \theta-1)^{2}-d_{2}^{2}\right] \leq \omega^{2} \leq k^{2}\left(s_{2}^{2}-d_{2}^{2}\right) .
$$


It is clear that the second inequality does not hold if $d_{2} \geq s_{2}$. Hence there is no $\omega>0$, implying that the delays are harmless in the two-delay dynamic model.

Theorem 6. If $s_{2} \leq d_{2}$, then the positive steady state of (29) is locally asymptotically stable for any positive values of $\tau_{1}$ and $\tau_{2}$.

On the other hand, if $s_{2}>d_{2}$, then conditions (45) hold for $\omega$ in the interval $\left[\omega_{s}, \omega_{e}\right]$, where

$$
\omega_{s}= \begin{cases}0 & \text { if } \theta \leq \theta_{0} \\ k \sqrt{s_{2}^{2}(2 \theta-1)^{2}-d_{2}^{2}} & \text { if } \theta>\theta_{0}\end{cases}
$$

with

$$
\begin{aligned}
& \theta_{0}=\frac{1}{2}\left(1+\frac{d_{2}}{s_{2}}\right) \quad \text { that solves } s_{2}^{2}(2 \theta-1)^{2}-d_{2}^{2}=0, \\
& \omega_{e}=k \sqrt{s_{2}^{2}-d_{2}^{2}},
\end{aligned}
$$

where the subscripts " $s$ " and " $e$ " mean the starting point and the end point, respectively.

We will next find all the pairs of $\left(\tau_{1}, \tau_{2}\right)$ satisfying $a(i \omega)=$ 0 . Let $|1|$ be the base of the triangle and then denote an angle between $|1|$ and $\left|a_{1}(i \omega)\right|$ by $\theta_{1}$ and an angle between $|1|$ and $\left|a_{2}(i \omega)\right|$ by $\theta_{2}$. By the law of cosine, the angle's magnitudes are expressed in terms of the model parameters:

$$
\begin{aligned}
& \theta_{1}(\omega)=\cos ^{-1}\left(\frac{\omega^{2}+\alpha^{2}+\beta^{2}-\gamma^{2}}{2 \beta \sqrt{\alpha^{2}+\omega^{2}}}\right), \\
& \theta_{2}(\omega)=\cos ^{-1}\left(\frac{\omega^{2}+\alpha^{2}-\beta^{2}+\gamma^{2}}{2 \gamma \sqrt{\alpha^{2}+\omega^{2}}}\right) .
\end{aligned}
$$

From (40), we obtain the arguments

$$
\begin{aligned}
& \arg \left[a_{1}(i \omega)\right]=-\tan ^{-1}\left(\frac{\omega}{\alpha}\right), \\
& \arg \left[a_{2}(i \omega)\right]=-\tan ^{-1}\left(\frac{\omega}{\alpha}\right) .
\end{aligned}
$$

Since the triangle can be located above and under the real axis, the following two equations hold for $\tau_{1}$ and $\tau_{2}$ :

$$
\begin{aligned}
& \left\{\arg \left[a_{1}(i \omega) e^{-i \omega \tau_{1}}\right]+2 m \pi\right\} \pm \theta_{1}(\omega)=\pi, \\
& \left\{\arg \left[a_{2}(i \omega) e^{-i \omega \tau_{2}}\right]+2 n \pi\right\} \mp \theta_{2}(\omega)=\pi
\end{aligned}
$$

which yield the threshold values of the delays

$$
\begin{aligned}
& \tau_{1}^{ \pm}(\omega, m)=\frac{1}{\omega}\left[-\tan ^{-1}\left(\frac{\omega}{\alpha}\right)+(2 m-1) \pi \pm \theta_{1}(\omega)\right], \\
& \tau_{2}^{\mp}(\omega, n)=\frac{1}{\omega}\left[-\tan ^{-1}\left(\frac{\omega}{\alpha}\right)+(2 n-1) \pi \mp \theta_{2}(\omega)\right] .
\end{aligned}
$$

Here $m$ and $n$ are nonnegative integers such that $\tau_{1}>0$ and $\tau_{2}>0$. Thus for any $m, n$, and $\omega \in\left[\omega_{s}, \omega_{e}\right]$, we can define the pairs of $\left(\tau_{1}, \tau_{2}\right)$ constructing the stability switching curve as follows.

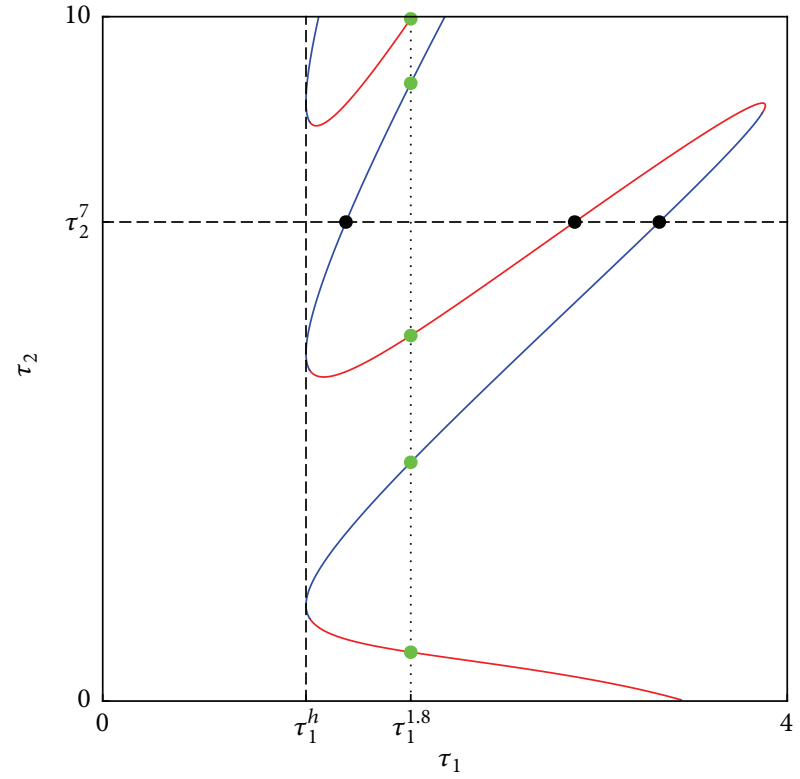

FIGURE 2: Stability switching curve.

Theorem 7. Given $s_{2}>d_{2}$, the stability switching curve is described by $C_{1}(m, n) \cup C_{2}(m, n)$ with $m, n=0,1,2, \ldots$, where

$$
\begin{aligned}
& C_{1}(m, n)=\left\{\tau_{1}^{+}(\omega, m), \tau_{2}^{-}(\omega, n)\right\}, \\
& C_{2}(m, n)=\left\{\tau_{1}^{-}(\omega, m), \tau_{2}^{+}(\omega, n)\right\} .
\end{aligned}
$$

The segments $C_{1}(m, n+1)$ and $C_{2}(m, n)$ have the same starting point whereas the segments $C_{1}(m, n)$ and $C_{2}(m, n)$ have the same end point.

Proof. It can be verified that

$$
\begin{aligned}
& \frac{\omega_{s}^{2}+\alpha^{2}+\beta^{2}-\gamma^{2}}{2 \beta \sqrt{\alpha^{2}+\omega_{s}^{2}}}=1, \\
& \frac{\omega_{e}^{2}+\alpha^{2}+\beta^{2}-\gamma^{2}}{2 \beta \sqrt{\alpha^{2}+\omega_{e}^{2}}}=1, \\
& \frac{\omega_{s}^{2}+\alpha^{2}-\beta^{2}+\gamma^{2}}{2 \gamma \sqrt{\alpha^{2}+\omega_{s}^{2}}}=-1, \\
& \frac{\omega^{2}+\alpha^{2}-\beta^{2}+\gamma^{2}}{2 \gamma \sqrt{\alpha^{2}+\omega^{2}}}=1
\end{aligned}
$$

which leads to

$$
\begin{aligned}
& \theta_{1}\left(\omega_{s}\right)=0, \\
& \theta_{1}\left(\omega_{e}\right)=0, \\
& \theta_{2}\left(\omega_{s}\right)=\pi, \\
& \theta_{2}\left(\omega_{e}\right)=0 .
\end{aligned}
$$




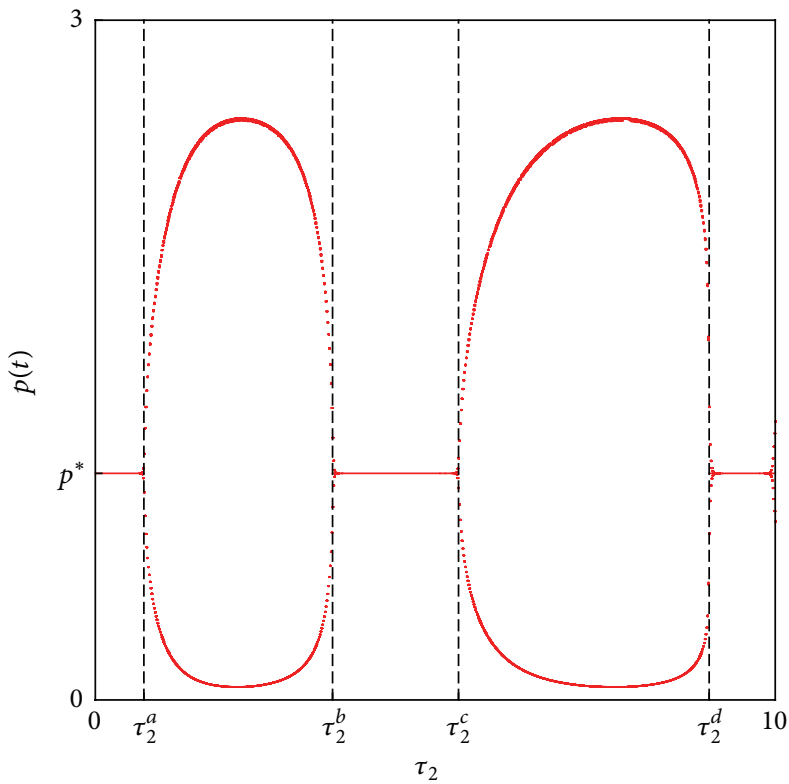

(a) $\tau_{1}=1.8$

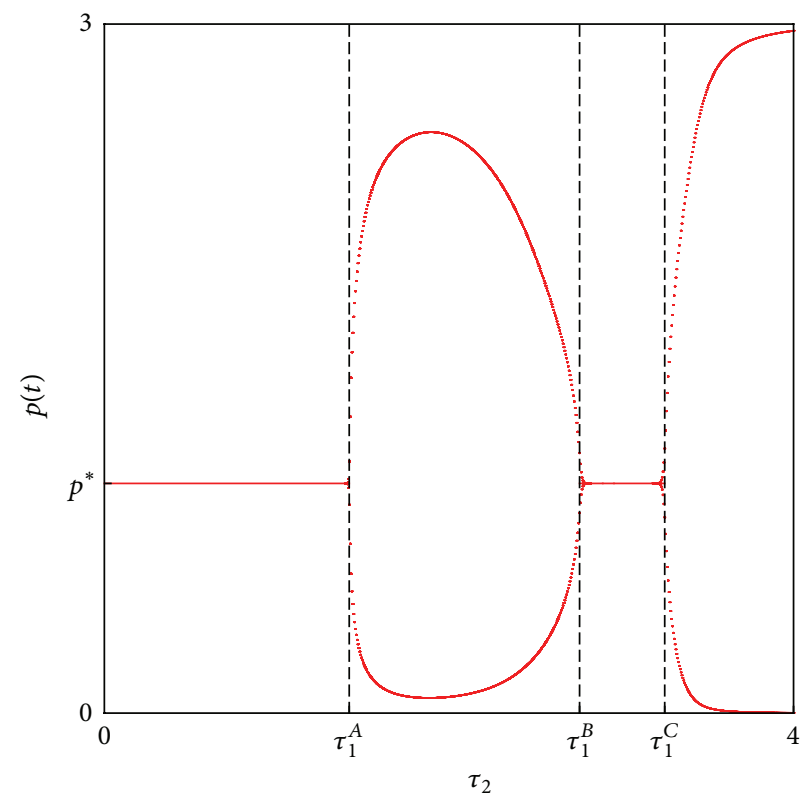

(b) $\tau_{2}=7$

FIGURE 3: Bifurcation diagrams showing stability losses and gains.

Let the starting points of $C_{1}(m, n)$ and $C_{2}(m, n)$ be denoted by

$$
\begin{aligned}
& C_{1}^{s}(m, n)=\left\{\tau_{1}^{+}\left(\omega_{s}, m\right), \tau_{2}^{-}\left(\omega_{s}, n\right)\right\}, \\
& C_{2}^{s}(m, n)=\left\{\tau_{1}^{-}\left(\omega_{s}, m\right), \tau_{2}^{+}\left(\omega_{s}, n\right)\right\} .
\end{aligned}
$$

Substituting $\theta_{1}\left(\omega_{s}\right)=0$ and $\theta_{2}\left(\omega_{s}\right)=\pi$ into (51) and (52) yields

$$
\begin{aligned}
\tau_{1}^{+}\left(\omega_{s}, m\right) & =\tau_{1}^{-}\left(\omega_{s}, m\right), \\
\tau_{2}^{-}\left(\omega_{s}, n+1\right) & =\tau_{2}^{+}\left(\omega_{s}, n\right)
\end{aligned}
$$

which imply that $C_{1}^{s}(m, n+1)=C_{2}^{s}(m, n)$. In the same way, let the end points of $C_{1}(m, n)$ and $C_{2}(m, n)$ be denoted by

$$
\begin{aligned}
& C_{1}^{e}(m, n)=\left\{\tau_{1}^{+}\left(\omega_{e}, m\right), \tau_{2}^{-}\left(\omega_{e}, n\right)\right\}, \\
& C_{2}^{e}(m, n)=\left\{\tau_{1}^{-}\left(\omega_{e}, m\right), \tau_{2}^{+}\left(\omega_{e}, n\right)\right\} .
\end{aligned}
$$

Then substituting $\theta_{1}\left(\omega_{e}\right)=0$ and $\theta_{2}\left(\omega_{e}\right)=0$ into (51) and (52) yields

$$
\begin{gathered}
\tau_{1}^{+}\left(\omega_{e}, m\right)=\tau_{1}^{-}\left(\omega_{e}, m\right), \\
\tau_{2}^{-}\left(\omega_{e}, n\right)=\tau_{2}^{+}\left(\omega_{e}, n\right)
\end{gathered}
$$

which imply that $C_{1}^{e}(m, n)=C_{2}^{e}(m, n)$. This completes the proof.

Under Assumption 3 with $\theta=0.8$ and $m=1$, the stability switching curve is illustrated in Figure 2 in which the red segments show $C_{1}(1, n)$ and the blue segments show $C_{2}(1, n)$ for $n=1,2,3$. Notice the segment shifts upward as the value of $n$ increases and to the right as the value of $m$ increases. ${ }^{4}$ The lowest red and blue segments are $C_{1}(1,1)$ and $C_{2}(1,1)$ where they are connected to each other at point $C_{1}^{e}(1,1)=C_{2}^{e}(1,1)$. The middle red and blue segments are $C_{1}(1,2)$ and $C_{2}(1,2)$ where $C_{1}(1,2)$ is connected to $C_{2}(1,1)$ at $C_{1}^{s}(1,2)=C_{2}^{s}(1,1)$. As $n$ increases the two segments are connected in the same way to construct the continuous stability switching curve, $C_{1}(1, n) \cup C_{2}(1, n)$. This curve divides the first quadrant into two parts as shown in Figure 2. One contains the origin and its every point can be reached from the origin via continuous curve not crossing the stability switching curve. At any point in this region, the real parts of the eigenvalues are negative, so the system is locally asymptotically stable. On the other hand, at the points in the complement of this region except the stability switching curve, the system is unstable. Observing Figure 2, we find the following three issues:

(i) For $\tau_{1} \leq \tau_{1}^{h} \simeq 1.188$, the system is locally asymptotically stable irrespective of the values of $\tau_{2}$, implying that delay $\tau_{2}$ is harmless.

(ii) For $\tau_{1}>\tau_{1}^{h}$, stability loss and gain repeatedly occur when $\tau_{2}$ increases from zero.

(iii) Depending on the value of $\tau_{2}$, two different dynamic phenomena are seen when the value of $\tau_{1}$ increases. One is when stability is lost and cannot be regained as in the one-delay model, and the other case is when stability regain can occur.

We now examine the effect caused by changing $\tau_{2}$, keeping the value of $\tau_{1}$ at some positive value. In Figure 3(a), the bifurcation diagram of $p(t)$ with respect to $\tau_{2}$ is illustrated. The value of $\tau_{1}$ is fixed at $\tau_{1}^{1.8}=1.8$ and the value of $\tau_{2}$ is increased along the vertical dotted line at $\tau_{1}^{1.8}$ in Figure 2 . 
For each value of $\tau_{2}$, the dynamic system runs for $0 \leq t \leq$ 1000 and discards the data for $0 \leq t \leq 950$ to get rid of the transients. The local maximum and minimum obtained from the remaining data are plotted against the value of $\tau_{2}$. The value of $\tau_{2}$ is increased with $1 / 400$ and then the same procedure is repeated until $\tau_{2}$ arrives at 10 . If the resultant bifurcation diagram has only one point against the value of $\tau_{2}$, then the system is locally stable and that point corresponds to the stationary point. If it has two points, then a limit cycle with one maximum and one minimum emerges. As is seen in Figure 2, the vertical line at $\tau_{1}=\tau_{1}^{1.8}$ crosses the stability switching curve five times. We denote the values of $\tau_{2}$ of the green intersection points by $\tau_{2}^{a}(\simeq 0.714), \tau_{2}^{b}(\simeq 3.489)$, $\tau_{2}^{c}(\simeq 5.342), \tau_{2}^{d}(\simeq 9.028)$, and $\tau_{2}^{e}(\simeq 9.928)$ in the ascending order. The bifurcation diagram in Figure 3(a) indicates the following dynamics: after stability is lost at $\tau_{2}^{a}$, a limit cycle emerges for $\tau_{2} \in\left(\tau_{2}^{a}, \tau_{2}^{b}\right)$ and its amplitude first expands and then shrinks to zero at $\tau_{2}=\tau_{2}^{b}$ when stability is regained. The same process is repeated for larger values of $\tau_{2}$.

We next draw attention to the effect caused by changing the value of $\tau_{1}$. With similar procedure, Figure 3(b) illustrates the bifurcation diagram with respect to $\tau_{1}$ along the dotted horizontal line at $\tau_{2}=\tau_{2}^{7}(=7)$ shown in Figure 2 in which the line crosses the stability switching line three times denoted by three black dots at $\tau_{1}^{A}(\simeq 1.421), \tau_{1}^{B}(\simeq 2.758)$, and $\tau_{1}^{C}(\simeq$ 3.252) in the ascending order. A limit cycle emerges when stability is lost and stability losses and gains are repeatedly observed.

\section{Two-Market Model with Two Delays}

In this section we examine two-delay price adjustments in the interrelated markets like hog and corn markets. Let us denote corn and hogs by $c$ and $h$. To simplify the analysis, the demand and supply functions in the corn market are assumed to be linear:

$$
\begin{aligned}
D^{c}\left(p_{c}(t)\right) & =\delta_{1}-\delta_{2} p_{c}(t), \\
S^{c}\left(p_{c}^{e}(t)\right) & =\sigma_{1}+\sigma_{2} p_{c}^{e}(t)
\end{aligned}
$$

and those in the hog market are also linear:

$$
\begin{aligned}
D^{h}\left(p_{h}(t)\right) & =d_{1}-d_{2} p_{h}(t), \\
S^{h}\left(p_{h}^{e}(t), p^{c}(t)\right) & =s_{1}+s_{2} p_{h}^{e}(t)-s_{3} p_{c}(t-\tau),
\end{aligned}
$$

where all parameters $\delta_{i}, \sigma_{i}, d_{i}, s_{i}$, and delay $\tau$ are positive. In each market, the demand for the commodity depends on its current price observed at time $t$. The supply of corn depends only on the expected corn price while the supply of hogs depends on the expected price of hogs and the delay price of corn since hog suppliers are corn demanders who determine their demand decisions observing the current price. It is natural for hog producers to have $s_{3}>0$ because they decrease the quantity of hogs when the corn price increases.
Equilibrium prices satisfying $D^{c}\left(p_{c}^{*}\right)=S^{c}\left(p_{c}^{*}\right)$ and $D^{h}\left(p_{h}^{*}\right)=$ $S^{h}\left(p_{h}^{*}, p_{c}^{*}\right)$ are

$$
\begin{aligned}
& p_{c}^{*}=\frac{\delta_{1}-\sigma_{1}}{\delta_{2}+\sigma_{2}}, \\
& p_{h}^{*}=\frac{d_{1}-s_{1}+s_{3} p_{c}^{*}}{d_{2}+s_{2}},
\end{aligned}
$$

where $\delta_{1}>\sigma_{1}$ and $d_{1}>s_{1}$ are assumed to assure positivity of the equilibrium prices. These conditions can also be interpreted by requiring that maximum demands are larger than minimum supplies. We assume the simplest expectation formations as in Assumption 1.

Assumption 8. Consider $p_{c}^{e}(t)=p_{c}\left(t-\tau_{c}\right)$ and $p_{h}^{e}(t)=p_{h}(t-$ $\tau_{h}$ ) with $\tau_{c}>0$ and $\tau_{h}>0$ and $\tau=\tau_{h}$.

The price adjustment system is given by a twodimensional system of delay differential equations:

$$
\begin{aligned}
& \dot{p}_{c}(t)=k_{c} p_{c}(t)\left[\left(\delta_{1}-\sigma_{1}\right)-\delta_{2} p_{c}(t)-\sigma_{2} p_{c}\left(t-\tau_{c}\right)\right], \\
& \dot{p}_{h}(t)=k_{h} p_{h}(t)\left[\left(d_{1}-s_{1}\right)-d_{2} p_{h}(t)-s_{2} p_{h}\left(t-\tau_{h}\right)\right. \\
& \left.\quad+s_{3} p_{c}\left(t-\tau_{h}\right)\right] .
\end{aligned}
$$

It can be confirmed that the equilibrium prices are the stationary point of the adjustment system. Linearizing this system in a neighborhood of the stationary point and introducing new variables and new parameters

$$
\begin{aligned}
x(t) & =p_{c}(t)-p_{c}^{*}, \\
y(t) & =p_{h}(t)-p_{h}^{*}, \\
\alpha & =k_{c} p_{c}^{*}, \\
\beta & =k_{h} p_{h}^{*}, \\
\tau_{x} & =\tau_{c}, \\
\tau_{y} & =\tau_{h}
\end{aligned}
$$

yield the linearized system

$$
\begin{aligned}
& \dot{x}(t)=\alpha\left[-\delta_{2} x(t)-\sigma_{2} x\left(t-\tau_{x}\right)\right], \\
& \dot{y}(t)=\beta\left[s_{3} x\left(t-\tau_{y}\right)-d_{2} y(t)-s_{2} y\left(t-\tau_{y}\right)\right] .
\end{aligned}
$$

Supposing exponential solutions $x(t)=e^{-\lambda t} u$ and $y(t)=$ $e^{-\lambda t} v$ with $u \neq 0$ and $v \neq 0$ and substituting them into (65), we see that nontrivial solutions for $u$ and $v$ exist if and only if

$$
\begin{aligned}
& \left(\begin{array}{cc}
\lambda+\alpha \delta_{2}+\alpha \sigma_{2} e^{-\lambda \tau_{x}} & 0 \\
-\beta s_{3} e^{-\lambda \tau_{y}} & \lambda+\beta d_{2}+\beta s_{2} e^{-\lambda \tau_{y}}
\end{array}\right)\left(\begin{array}{l}
u \\
v
\end{array}\right) \\
& =\left(\begin{array}{l}
0 \\
0
\end{array}\right) .
\end{aligned}
$$

The corresponding characteristic equation is

$$
\left(\lambda+\alpha \delta_{2}+\alpha \sigma_{2} e^{-\lambda \tau_{x}}\right)\left(\lambda+\beta d_{2}+\beta s_{2} e^{-\lambda \tau_{y}}\right)=0
$$


which implies that the two delays are independent. ${ }^{5}$ Notice that the delay corn price $p_{c}\left(t-\tau_{h}\right)$ does not affect local dynamics. Equation (67) can be divided into two independent equations:

$$
\begin{aligned}
& \lambda+\alpha \delta_{2}+\alpha \sigma_{2} e^{-\lambda \tau_{x}}=0, \\
& \lambda+\beta d_{2}+\beta s_{2} e^{-\lambda \tau_{y}}=0 .
\end{aligned}
$$

Before proceeding we check the no delay case (i.e., $\tau_{x}=\tau_{y}=$ $0)$ in which, from (67), the characteristic roots are real and negative:

$$
\begin{aligned}
& \lambda=-\alpha\left(\delta_{2}+\sigma_{2}\right)<0, \\
& \lambda=-\beta\left(d_{2}+s_{2}\right)<0 .
\end{aligned}
$$

The stationary point is always stable without delays. Thus stability can be preserved for positive delays as far as the delays are sufficiently small. In order to confirm to what extent the stationary point is stable, we determine the threshold values of the delays for which stability is just lost. Equations (68) and (69) have the same form as (16) and can be solved in the same way. Substituting $\lambda=i \omega_{x}$ and $\lambda=i \omega_{y}$ into (68) and (69) yields

$$
\begin{aligned}
& i \omega_{x}+\alpha \delta_{2}+\alpha \sigma_{2}\left(\cos \tau_{x} \omega_{x}-\sin \tau_{x} \omega_{x}\right)=0, \\
& i \omega_{y}+\beta d_{2}+\beta s_{2}\left(\cos \tau_{y} \omega_{y}-\sin \tau_{y} \omega_{y}\right)=0 .
\end{aligned}
$$

Dividing each equation into the real and imaginary parts and solving them for $\omega_{j}$ for $j=x, y$, we have

$$
\begin{aligned}
& \omega_{x}^{*}=\alpha \sqrt{\sigma_{2}^{2}-\delta_{2}^{2}}, \\
& \omega_{y}^{*}=\beta \sqrt{s_{2}^{2}-d_{2}^{2}} .
\end{aligned}
$$

There are several combinations of $\omega_{x}$ and $\omega_{y}$ according to whether $\sigma_{2}-\delta_{2}$ and $s_{2}-d_{2}$ are positive or not. To simplify the analysis, we assume the following.

Assumption 9. Consider $\sigma_{2}-\delta_{2}>0$ and $s_{2}-d_{2}>0$.

These conditions mean that supplies increase faster than demands decrease when market or expected price increases.

Under Assumption 9 we can determine the threshold values of the delays ${ }^{6}$

$$
\begin{aligned}
& \tilde{\tau}_{x, m}=\frac{1}{\omega_{x}^{*}}\left[\cos ^{-1}\left(-\frac{\delta_{2}}{\sigma_{2}}\right)+2 m \pi\right], \\
& \tilde{\tau}_{y, n}=\frac{1}{\omega_{y}^{*}}\left[\cos ^{-1}\left(-\frac{d_{2}}{s_{2}}\right)+2 n \pi\right] .
\end{aligned}
$$

At $\tau_{j}=\tilde{\tau}_{j, 0}$ for $j=x, y$, the stationary point loses stability. Hence the $\tau_{x}=\tilde{\tau}_{x, 0}$ line and the $\tau_{y}=\tilde{\tau}_{y, 0}$ line form the stability switching curve. Returning to the original notation of the delays and using these lines, we divide the $\left(\tau_{c}, \tau_{h}\right)$ plane into four parts as shown in Figure 4 . The stationary point is locally asymptotically stable in the yellow region in which

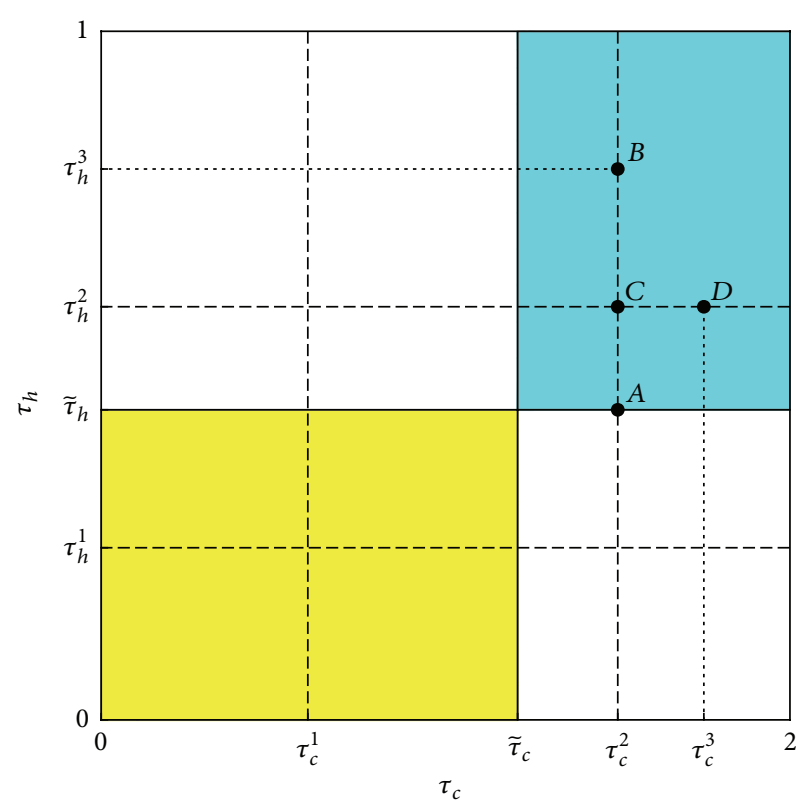

FIGURE 4: Stability switching curve.

$0 \leq \tau_{c} \leq \widetilde{\tau}_{c}$ and $0 \leq \tau_{h} \leq \widetilde{\tau}_{h}$ and unstable otherwise (in the union of the white regions and the blue region) where $\tilde{\tau}_{c}=\tilde{\tau}_{x, 0}$ and $\tilde{\tau}_{h}=\tilde{\tau}_{y, 0}$. More precisely, one of the two equations in (65) is unstable in the white regions and the two equations are unstable in the blue region. To see the effects caused by the delays, we perform simulations under the following numerical specification.

Assumption 10. Consider $\delta_{1}=4, \delta_{2}=1, \sigma_{1}=1, \sigma_{2}=2$, $d_{1}=4, d_{2}=1, s_{1}=1, s_{2}=3$, and $s_{3}=2$.

Notice that under these conditions maximum demands are larger than minimum supplies, so the equilibrium is positive. Furthermore Assumption 9 is also satisfied, so stability switches can be observed.

Under Assumption 10, the threshold values of the delays are

$$
\begin{aligned}
& \tilde{\tau}_{c}=\frac{2 \pi}{2 \sqrt{3}} \simeq 1.209, \\
& \tilde{\tau}_{h}=\frac{\cos ^{-1}(-1 / 3)}{3 \sqrt{2}} \simeq 0.450 .
\end{aligned}
$$

Fixing the value of $\tau_{c}$ at $\tau_{c}^{1}=0.6$ in the first simulation and $\tau_{c}^{2}=1.5$ in the second simulation, we investigate the delay effect caused by changing the value of $\tau_{h}$. The simulations are performed in the same way as before. The value of $\tau_{h}$ is increased from 0 to 1 with an increment of $1 / 400$ and the delay model (63) is run for $0 \leq t \leq 500$ for each value of $\tau_{h}$. The results obtained are summarized in Figures 5(a) and 5(b) in which the bifurcation diagrams of $p_{h}$ are illustrated. In the first simulation, the stationary state is locally stable for $\tau_{h}<\widetilde{\tau}_{h}$ while at $\tau_{h}=\tilde{\tau}_{h}$ the real part of one eigenvalue becomes positive from negative and thus stability is lost. It bifurcates to a limit cycle and no stability regain occurs for further 


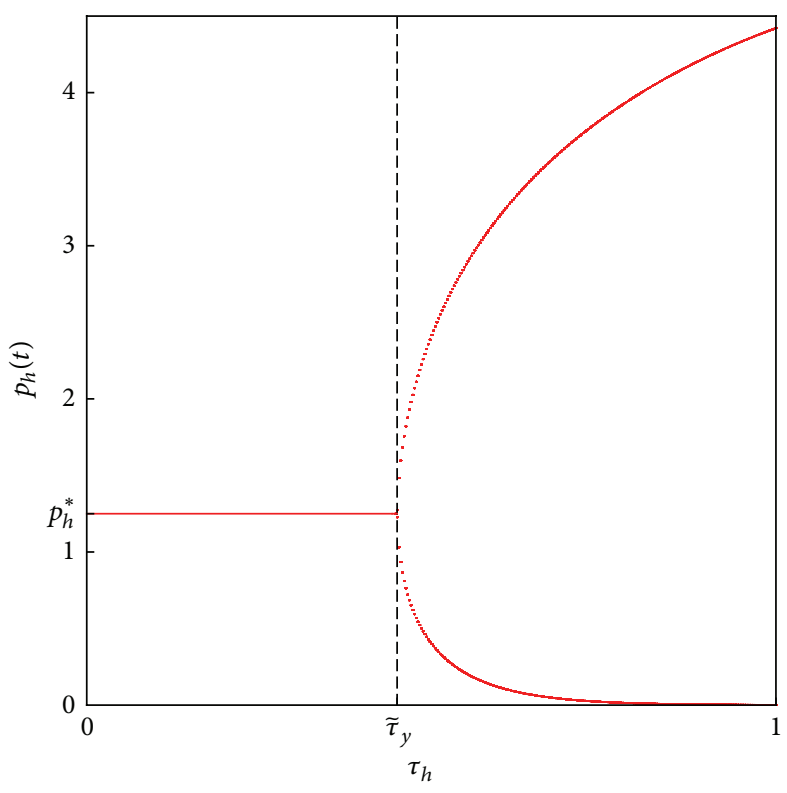

(a) $\tau_{c}=\tau_{c}^{1}=0.6$

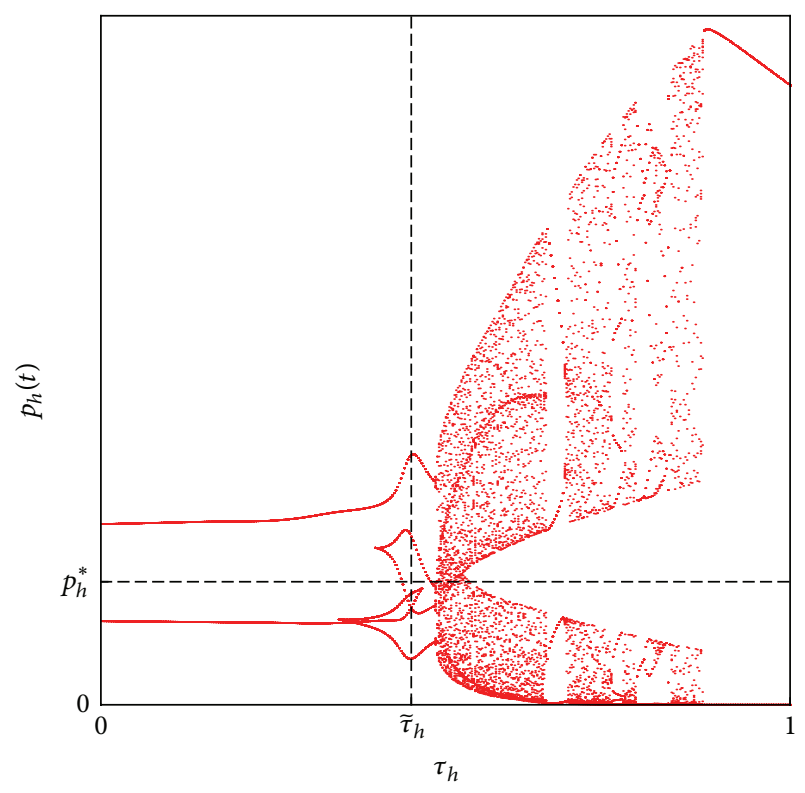

(b) $\tau_{c}=\tau_{c}^{2}=1.5$

FIgURE 5: Bifurcation diagrams with respect to $\tau_{h}$.

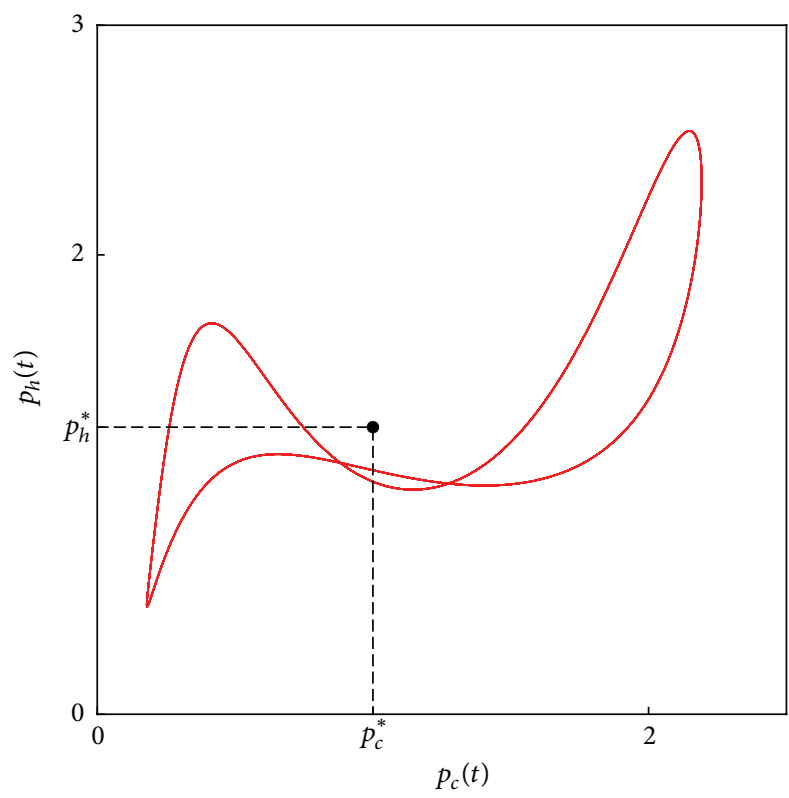

(a) $\tau_{c}=\tau_{c}^{2}$ and $\tau_{h}=\widetilde{\tau}_{h}$

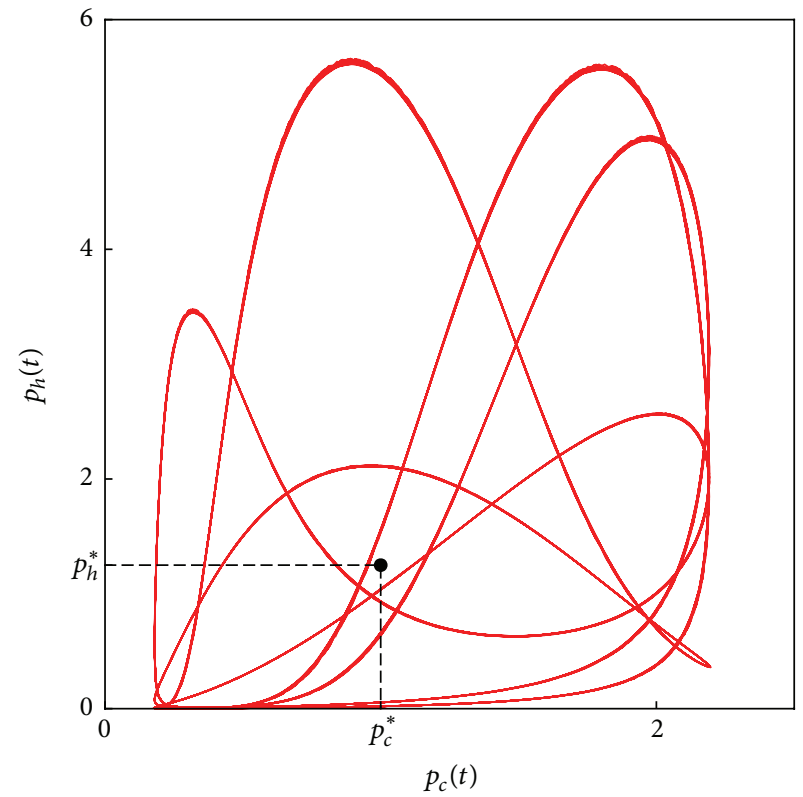

(b) $\tau_{c}=\tau_{c}^{2}$ and $\tau_{h}=\tau_{h}^{3}$

Figure 6: Phase diagrams at points $A$ and $B$.

increasing $\tau_{h}$ from $\tilde{\tau}_{h}$. This result is essentially the same as the one obtained in the single delay model. On the other hand, in the second simulation, we obtain qualitatively different results. The stationary state is unstable even for $\tau_{h}=0$ since $\tau_{c}^{2}>\widetilde{\tau}_{c}$. The bifurcation diagram in Figure 5(b) indicates that a limit cycle already emerges for $\tau_{h}=0$, it is distorted for $\tau_{h}$ close to $\widetilde{\tau}_{h}$, and further increasing $\tau_{h}$ generates complicated dynamics.

Figure 6 provides two phase diagrams of $p_{c}(t)$ and $p_{h}(t)$ for points $A$ and $B$ in Figure 4 . It is seen in Figure 5(b) that the vertical dotted line at $\tau_{h}=\widetilde{\tau}_{h}$ crosses the bifurcation diagram six times. This phenomenon is described from a different viewpoint in Figure 6(a) in which the distorted limit cycle has three local maximums and three local minimums for point $A=\left(\tau_{c}^{2}, \widetilde{\tau}_{h}\right)$. The value of $\tau_{h}$ is then increased to $\tau_{c}^{3}=0.6$ while $\tau_{c}$ is kept at the same value. It is seen in Figure 6(b) that the limit cycle has seven local maximums and minimums if we observe it carefully and thus its time trajectory exhibits fluctuations with more ups and downs. 


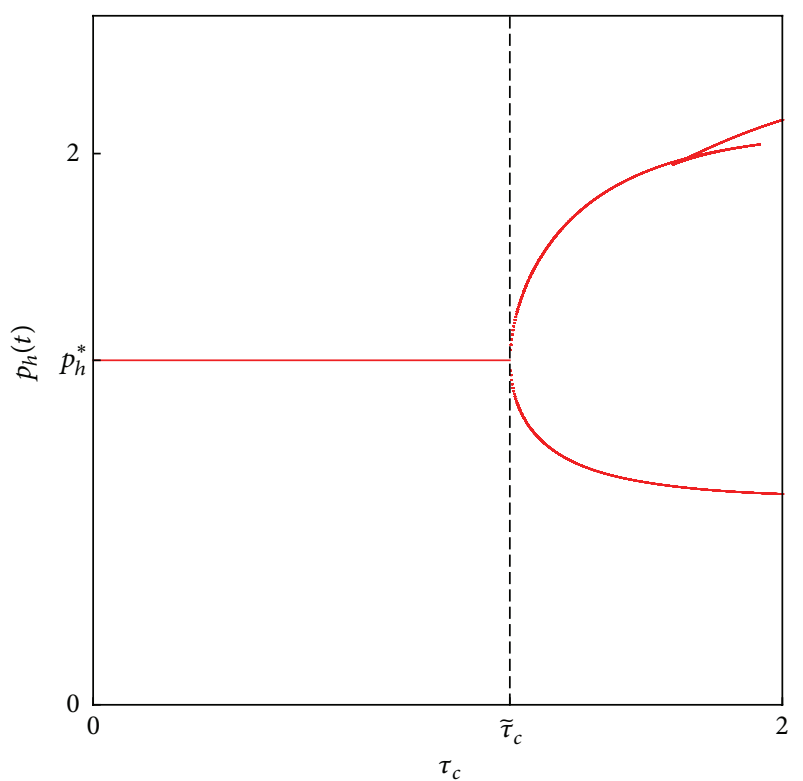

(a) $\tau_{h}^{A}=0.25$

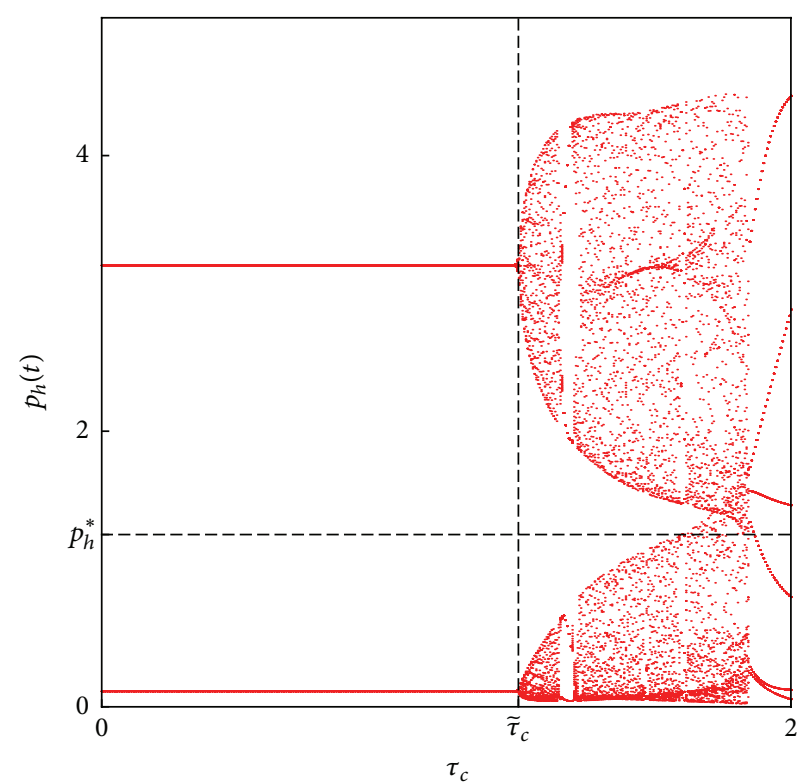

(b) $\tau_{h}^{B}=0.6$

FIGURE 7: Bifurcation diagram with respect to $\tau_{c}$.

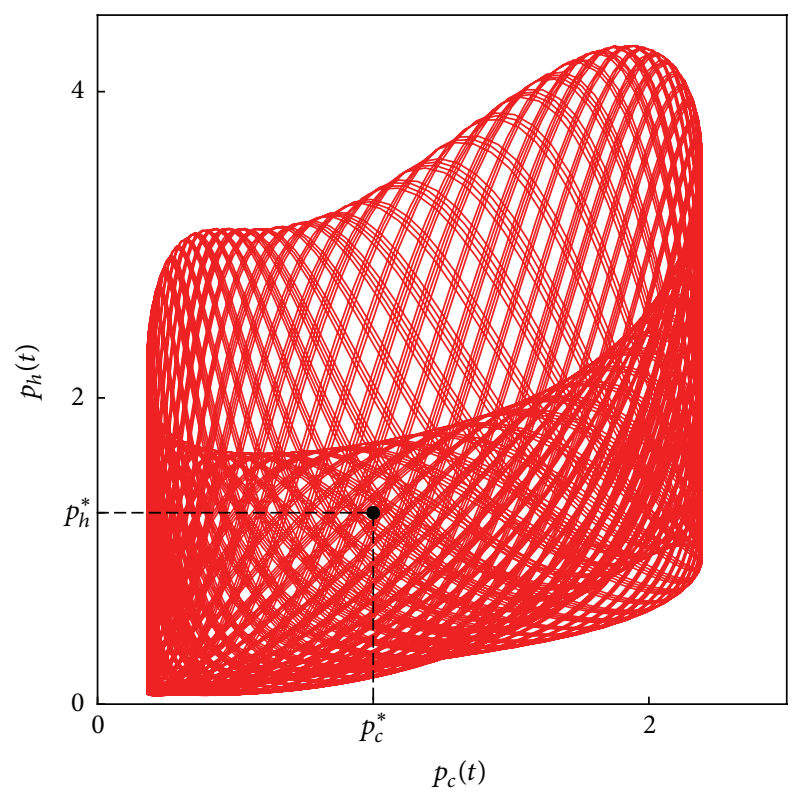

(a) $\tau_{c}=\tau_{c}^{2}$ and $\tau_{h}=\tau_{h}^{2}$

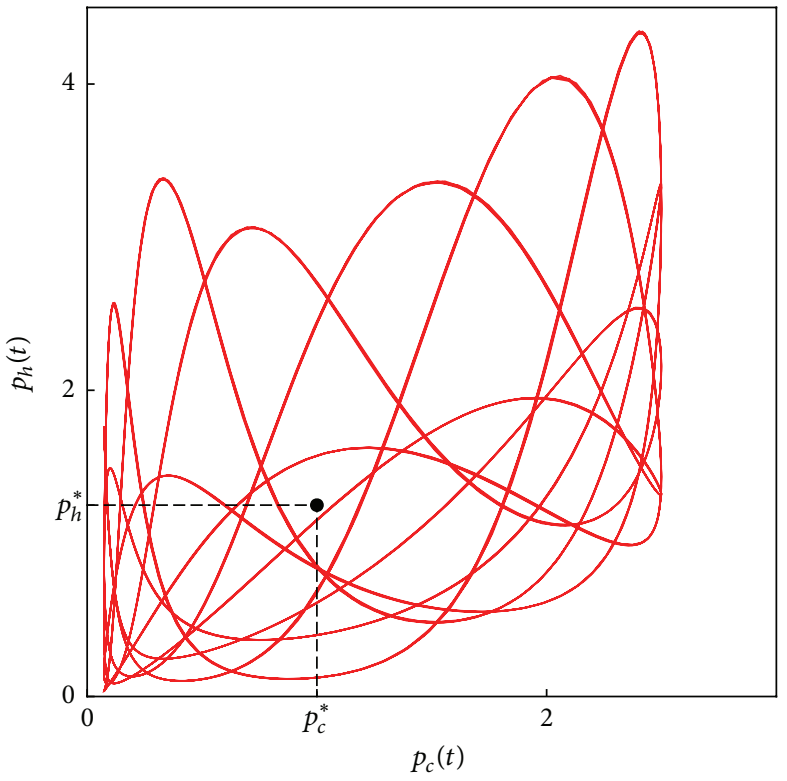

(b) $\tau_{c}=\tau_{c}^{3}$ and $\tau_{h}=\tau_{h}^{2}$

Figure 8: Phase diagrams at points $C$ and $D$.

Further we examine the effects caused by changing the value of $\tau_{c}$. For this purpose, fixing the value of $\tau_{h}$ at $\tau_{h}^{1}=$ 0.25 in the first simulation and at $\tau_{h}^{2}=0.6$ in the second simulation, we increase the value of $\tau_{c}$ from 0 to 2 with $1 / 400$ increment and run delay model (63) for each value of $\tau_{c}$. When the value of $\tau_{h}$ is chosen in the yellow (i.e., stable) region, the dynamic system generates simple dynamics as shown in Figure 7(a) in which the system is stable for $\tau_{c}<$ $\tilde{\tau}_{c}$ and gives rise to a limit cycle after stability is lost for $\tau_{c}>\tilde{\tau}_{c}$. As expected, when both equations in (63) are destabilized, more complicated dynamics can arise as shown in Figure 7(b).

We now shift our emphasis from the bifurcation diagrams to the phase diagrams, especially to see what dynamics arise when both equations of delay system (63) are locally unstable. In Figure 8(a), we choose point $C$ in Figure 4 and run the system for $0 \leq t \leq 500$. We eliminate the price data for $t \leq 300$ as transitory dynamics and plot the remaining data 
in the $\left(p_{c}, p_{h}\right)$ plane. It can be seen that the prices behave in a very complicated way. In Figure $8(\mathrm{~b})$, the value of $\tau_{h}$ is increased to $\tau_{h}^{3}$. It is also seen that the price behavior is also complicated. What these numerical examples make clear is the following:

(i) If one equation of the two-delay system is stable, then the resultant dynamics is essentially the same as in the one-delay system.

(ii) If both equations are unstable, then the two-delay system can generate various dynamics from a simple limit cycle to complicated dynamics having many ups and downs.

\section{Concluding Remarks}

In this study, we have examined the delay effect on price dynamics in three different models. After reviewing a single delay model in which a limit cycle can emerge via Hopf bifurcation, we proceed to two models with two production delays. When the two delays are used to form an expected price or feedback for price adjustment, we find that the stability switching curve on which stability is changed is winding and as a natural consequence stability losses and gains are repeated when the length of one delay increases. It is numerically confirmed that only simple dynamics such as a limit cycle can emerge when stability is lost. We determine the stability switching curve under Assumption 3 and $\theta=$ 0.8 ; however other parameter specifications might result in different shapes of this curve. Gori et al. [15] investigated earlier how the structure of a two-delay model changes under variation of model parameters. In our next project we will examine the different shapes of the stability switching curves as the values of the parameters of our model vary.

On the other hand, when the two delays are considered in interrelated markets, they affect price dynamics differently. The stability switching curves become straight lines regardless of the values of the model parameters. When one market is stable and the other market is unstable, the resultant dynamics is simple and essentially the same as the one in the single delay model. However, when both markets are unstable, a broad spectrum of dynamic behaviors can be found. In the cobweb literature, the discrete-time model has been considered and it is known that it can give rise to complicated dynamics when behavior nonlinearities get stronger. This study develops a continuous-time model and indicates that it also reasonably explains various dynamic behaviors observed in commodity markets.

\section{Appendix}

In this Appendix, we apply the method developed by Lin and Wang [14] to solve a two-delay differential equation to our model. To this end, we first rewrite the left-hand side of (67):

$$
D\left(\lambda, \tau_{x}, \tau_{y}\right)=D_{x}\left(\lambda, \tau_{x}\right) D_{y}\left(\lambda, \tau_{y}\right)
$$

where

$$
\begin{aligned}
& D_{x}\left(\lambda, \tau_{x}\right)=q_{0}(\lambda)+q_{1}(\lambda) e^{-\lambda \tau_{x}}, \\
& D_{y}\left(\lambda, \tau_{y}\right)=q_{0}^{\prime}(\lambda)+q_{1}^{\prime}(\lambda) e^{-\lambda \tau_{y}}
\end{aligned}
$$

and $q_{j}(\lambda)$ and $q_{j}^{\prime}(\lambda)$ for $j=0,1$ are defined accordingly. Substituting (A.3) and (A.2) into (A.1) and expanding it yield the characteristic equation

$$
\begin{aligned}
D\left(\lambda, \tau_{x}, \tau_{y}\right)= & P_{0}(\lambda)+P_{1}(\lambda) e^{-\lambda \tau_{x}}+P_{2}(\lambda) e^{-\lambda \tau_{y}} \\
& +P_{3}(\lambda) e^{-\lambda\left(\tau_{x}+\tau_{y}\right)}
\end{aligned}
$$

where $P_{0}(\lambda)=q_{0}(\lambda) q_{0}^{\prime}(\lambda), P_{1}(\lambda)=q_{0}^{\prime}(\lambda) q_{1}(\lambda), P_{2}(\lambda)=$ $q_{0}(\lambda) q_{1}^{\prime}(\lambda)$, and $P_{3}(\lambda)=q_{1}(\lambda) q_{1}^{\prime}(\lambda)$. Since $\lambda=0$ is not a solution of $D\left(\lambda, \tau_{x}, \tau_{y}\right)=0$, we look for a pair of the delays for which the characteristic equation has purely imaginary roots. Since roots of a real function come in conjugate pairs, we can assume that $\lambda=i \omega$ and $\omega>0$. Substituting this into $D\left(\lambda, \tau_{x}, \tau_{y}\right)=0$, we have two different forms:

$$
\begin{aligned}
& \quad\left[P_{0}(i \omega)+P_{1}(i \omega) e^{-\lambda \tau_{x}}\right] \\
& \quad+\left[P_{2}(i \omega)+P_{3}(i \omega) e^{-\lambda \tau_{x}}\right] e^{-\lambda \tau_{y}}=0 \\
& {\left[P_{0}(i \omega)+P_{2}(i \omega) e^{-\lambda \tau_{y}}\right]} \\
& \quad+\left[P_{1}(i \omega)+P_{3}(i \omega) e^{-\lambda \tau_{y}}\right] e^{-\lambda \tau_{x}}=0 .
\end{aligned}
$$

We introduce new functions:

$$
\begin{aligned}
& A_{1}(\omega)=\operatorname{Re}\left[P_{2} \bar{P}_{3}-P_{0} \bar{P}_{1}\right], \\
& B_{1}(\omega)=\operatorname{Im}\left[P_{2} P_{3}-P_{0} \bar{P}_{1}\right], \\
& A_{2}(\omega)=\operatorname{Re}\left[P_{1} \bar{P}_{3}-P_{0} \bar{P}_{2}\right], \\
& B_{2}(\omega)=\operatorname{Im}\left[P_{1} \bar{P}_{3}-P_{0} \bar{P}_{2}\right],
\end{aligned}
$$

where

$$
\begin{aligned}
& P_{2} \bar{P}_{3}-P_{0} \bar{P}_{1}=q_{0} \bar{q}_{1}\left[q_{1}^{\prime} \bar{q}_{1}^{\prime}-q_{0}^{\prime} \bar{q}_{0}^{\prime}\right], \\
& P_{1} \bar{P}_{3}-P_{0} \bar{P}_{2}=q_{0}^{\prime} \bar{q}_{1}^{\prime}\left[q_{1} \bar{q}_{1}-q_{0} \bar{q}_{0}\right] .
\end{aligned}
$$

Following Lin and Wang [14], we have

$$
\begin{gathered}
\left|P_{0}(i \omega)\right|^{2}+\left|P_{1}(i \omega)\right|^{2}-\left|P_{2}(i \omega)\right|^{2}-\left|P_{3}(i \omega)\right|^{2} \\
=2 A_{1}(\omega) \cos \left(\omega \tau_{x}\right)-2 B_{1}(\omega) \sin \omega \tau_{x}, \\
\left|P_{0}(i \omega)\right|^{2}-\left|P_{1}(i \omega)\right|^{2}+\left|P_{2}(i \omega)\right|^{2}-\left|P_{3}(i \omega)\right|^{2} \\
\quad=2 A_{2}(\omega) \cos \left(\omega \tau_{y}\right)-2 B_{2}(\omega) \sin \omega \tau_{y} .
\end{gathered}
$$


Using (A.2) and (A.3), we obtain

$$
\begin{aligned}
& \left|P_{0}(i \omega)\right|^{2}+\left|P_{1}(i \omega)\right|^{2}-\left|P_{2}(i \omega)\right|^{2}-\left|P_{3}(i \omega)\right|^{2} \\
& =\left(\left|q_{0}\right|^{2}+\left|q_{1}\right|^{2}\right)\left(\left|q_{0}^{\prime}\right|^{2}-\left|q_{1}^{\prime}\right|^{2}\right), \\
& \left|P_{0}(i \omega)\right|^{2}-\left|P_{1}(i \omega)\right|^{2}+\left|P_{2}(i \omega)\right|^{2}-\left|P_{3}(i \omega)\right|^{2} \\
& =\left(\left|q_{0}^{\prime}\right|^{2}+\left|q_{1}^{\prime}\right|^{2}\right)\left(\left|q_{0}\right|^{2}-\left|q_{1}\right|^{2}\right) .
\end{aligned}
$$

We then finally arrive at the following forms:

$$
\begin{aligned}
& \left(\left|q_{0}\right|^{2}+\left|q_{1}\right|^{2}\right)\left(\left|q_{0}^{\prime}\right|^{2}-\left|q_{1}^{\prime}\right|^{2}\right) \\
& =2\left\{\operatorname{Re}\left[q_{0} \bar{q}_{1}\right]\left(\left|q_{1}^{\prime}\right|^{2}-\left|q_{0}^{\prime}\right|^{2}\right) \cos \omega \tau_{x}\right. \\
& \left.-\operatorname{Im}\left[q_{0} \bar{q}_{1}\right]\left(\left|q_{1}^{\prime}\right|^{2}-\left|q_{0}^{\prime}\right|^{2}\right) \sin \omega \tau_{x}\right\}, \\
& \left(\left|q_{0}^{\prime}\right|^{2}+\left|q_{1}^{\prime}\right|^{2}\right)\left(\left|q_{0}\right|^{2}-\left|q_{1}\right|^{2}\right) \\
& =2\left\{\operatorname{Re}\left[q_{0}^{\prime} \bar{q}_{1}^{\prime}\right]\left(\left|q_{1}\right|^{2}-\left|q_{0}\right|^{2}\right) \cos \omega \tau_{y}\right. \\
& \left.-\operatorname{Im}\left[q_{0}^{\prime} \bar{q}_{1}^{\prime}\right]\left(\left|q_{1}\right|^{2}-\left|q_{0}\right|^{2}\right) \sin \omega \tau_{y}\right\} .
\end{aligned}
$$

It is clear that if $\left|q_{0}^{\prime}\right|^{2}=\left|q_{1}^{\prime}\right|^{2}$ and $\left|q_{0}\right|^{2}=\left|q_{1}\right|^{2}$, then (A.10) and (A.11) hold for any $\tau_{x} \geq 0$ and $\tau_{y} \geq 0$, respectively. Further the conditions $\left|q_{0}^{\prime}\right|^{2}=\left|q_{1}^{\prime}\right|^{2}$ and $\left|q_{0}\right|^{2}=\left|q_{1}\right|^{2}$ can be rewritten as

$$
\begin{aligned}
& \omega_{y}^{*}=\beta \sqrt{s_{2}^{2}-d_{2}^{2}}, \\
& \omega_{x}^{*}=\alpha \sqrt{\sigma_{2}^{2}-\delta_{2}^{2}} .
\end{aligned}
$$

Notice that (A.12) is the same as (72). Let $\tau_{x}^{*}$ and $\tau_{y}^{*}$ be solutions of $D_{x}\left(i \omega_{x}^{*}, \tau_{x}\right)=0$ and $D_{y}\left(i \omega_{y}^{*}, \tau_{x}\right)=0$. These are equivalent to $\tilde{\tau}_{x, 0}$ and $\tilde{\tau}_{y, 0}$. Hence we obtain

$$
\begin{array}{ll}
D\left(i \omega_{x}^{*}, \tau_{x}^{*}, \tau_{y}\right)=0 & \text { holds for any } \tau_{y} \geq 0, \\
D\left(i \omega_{y}^{*}, \tau_{x}, \tau_{y}^{*}\right)=0 & \text { holds for any } \tau_{x} \geq 0 .
\end{array}
$$

On the other hand, if $\omega_{y} \neq \omega_{y}^{*}$ or $\left|q_{0}^{\prime}\right|^{2}-\left|q_{1}^{\prime}\right|^{2} \neq 0$, then from (A.10),

$$
\begin{aligned}
& \left|q_{0}\right|^{2}+\left|q_{1}\right|^{2} \\
& =2\left(\operatorname{Re}\left[q_{0} \bar{q}_{1}\right] \cos \omega \tau_{x}-\operatorname{Im}\left[q_{0} \bar{q}_{1}\right] \sin \omega \tau_{x}\right) \\
& \leq 2 \sqrt{\operatorname{Re}\left[q_{0} \bar{q}_{1}\right]^{2}+\operatorname{Im}\left[q_{0} \bar{q}_{1}\right]^{2}} \sqrt{\cos ^{2} \omega \tau_{x}+\sin ^{2} \omega \tau_{x}} \\
& =2\left|q_{0} \bar{q}_{1}\right|=2\left|q_{0}\right|\left|q_{1}\right|
\end{aligned}
$$

implying that $\left(\left|q_{0}\right|-\left|q_{1}\right|\right)^{2} \leq 0$ so $\left|q_{0}\right|=\left|q_{1}\right|$ meaning that in this case $\omega_{x}=\omega_{x}^{*}$ and $\omega_{y}$ is arbitrary. A similar argument shows that if $\omega_{x} \neq \omega_{x}^{*}$ or $\left|q_{0}\right|^{2}-\left|q_{1}\right|^{2} \neq 0$, then $\omega_{y}=\omega_{y}^{*}$ and $\omega_{x}$ is arbitrary. So there are no additional stability switching points besides the two lines $\tau_{x}=\tau_{x}^{*}$ and $\tau_{y}=\tau_{y}^{*}$.

In summary the stability switching curves are given by the two line segments as depicted in Figure 4:

$$
\begin{aligned}
& \tau_{x}=\tau_{x}^{*}, \\
& \tau_{y}=\tau_{y}^{*} .
\end{aligned}
$$

\section{Conflict of Interests}

The authors declare that there is no conflict of interests regarding the publication of this paper.

\section{Acknowledgments}

The authors are grateful to anonymous referees for their helpful comments. They also highly appreciate the financial supports from the MEXT-Supported Program for the Strategic Research Foundation at Private Universities 20132017, Japan Society for the Promotion of Science (Grantin-Aid for Scientific Research (C) 24530202, 25380238, and 26380316), and Chuo University (Joint Research Grant). The usual disclaimers apply.

\section{Endnotes}

1. Although dynamic equation (6) has also zero as stationary point, we ignore it in this study.

2. The stability condition in a continuous-time model is the same as the one in a discrete-time model. Assuming $\tau=$ 1, we can construct a discrete-time cobweb model:

$$
p(t)=-\frac{s_{2}}{d_{2}} p(t-1)+\left(d_{1}-s_{1}\right),
$$

where $s_{2} \leq d_{2}$ is the stability condition including a cyclic solution.

3. It is possible to substitute it into (18) to obtain the same value in a different form:

$$
\bar{\tau}=\frac{1}{\bar{\omega}}\left[\pi-\sin ^{-1}\left(-\frac{\bar{\omega}}{\beta}\right)\right] .
$$

4. Since the stability switching curve with $m=0$ is located in the second quadrant of the $\left(\tau_{1}, \tau_{2}\right)$ plane and the curve with $n=0$ is in the fourth quadrant, they are not depicted in Figure 2.

5. In the Appendix, we apply a more general method to solve the two-delay differential equation for this particular case and obtain the same result.

6. We can have the different form for the same threshold value:

$$
\begin{aligned}
& \tilde{\tau}_{x, m}=\frac{1}{\omega_{x}^{*}}\left[\pi-\sin ^{-1}\left(\frac{\omega_{x}^{*}}{\alpha \sigma_{2}}\right)+2 m \pi\right], \\
& \tilde{\tau}_{y, m}=\frac{1}{\omega_{y}^{*}}\left[\pi-\sin ^{-1}\left(\frac{\omega_{y}^{*}}{\beta s_{2}}\right)+2 n \pi\right] .
\end{aligned}
$$




\section{References}

[1] N. Kaldor, "A classificatory note on the determinateness of equilibrium," Review of Economic Studies, vol. 1, pp. 122-136, 1934.

[2] G. I. Bischi, C. Chiarella, M. Kopel, and F. Szidarovszky, NonLinear Oligopolies: Stability and Bifurcations, Springer, Berlin, Germany, 2010.

[3] K. Shirai and Y. Amano, "Nonlinear characteristics of the rate of return in the production process," International Journal of Innovative Computing, Information and Control, vol. 10, no. 2, pp. 601-616, 2014.

[4] R. Dieci and F. Westerhoff, "Interacting cobweb markets," Journal of Economic Behavior and Organization, vol. 75, no. 3, pp. 461-481, 2010.

[5] C. H. Hommes, "Dynamics of the cobweb model with adaptive expectations and nonlinear supply and demand," Journal of Economic Behavior \& Organization, vol. 24, no. 3, pp. 315-335, 1994.

[6] J. B. Haldane, "A contribution to the theory of price fluctuations," Review of Economic Studies, vol. 1, no. 3, pp. 186-195, 1934.

[7] A. B. Larson, "The hog cycle as harmonic motion," Journal of Farm Economics, vol. 46, no. 2, pp. 375-386, 1964.

[8] M. C. Mackey, "Commodity price fluctuations: price dependent delays and nonlinearities as explanatory factors," Journal of Economic Theory, vol. 48, no. 2, pp. 497-509, 1989.

[9] L. Gori, L. Guerrini, and M. Sodini, "Hopf bifurcation in a cobweb model with discrete time delays," Discrete Dynamics in Nature and Society, vol. 2014, Article ID 137090, 8 pages, 2014.

[10] R. Bellman and K. L. Cooke, Differential-Difference Equations, The Rand Corporation, Santa Monica, Calif, USA, 1963.

[11] A. Matsumoto and F. Szidarovszky, "An elementary study of a class of dynamic systems with single time delay," Cubo, vol. 15, no. 3, pp. 1-7, 2013.

[12] A. Matsumoto and F. Szidarovszky, "An elementary study of a class of dynamic systems with two time delays," Cubo, vol. 14, no. 3, pp. 103-113, 2012.

[13] K. Gu, S.-I. Niculescu, and J. Chen, "On stability crossing curves for general systems with two delays," Journal of Mathematical Analysis and Applications, vol. 311, no. 1, pp. 231-253, 2005.

[14] X. Lin and H. Wang, "Stability analysis of delay differential equations with two discrete delays," Canadian Applied Mathematics Quarterly, vol. 20, no. 4, pp. 519-533, 2012.

[15] L. Gori, L. Guerrini, and M. Sodini, "Hopf bifurcation and stability crossing curves in a cobweb model with heterogeneous producers and time delays," Nonlinear Analysis: Hybrid Systems, vol. 18, pp. 117-133, 2015. 


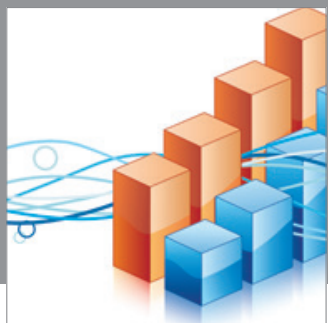

Advances in

Operations Research

mansans

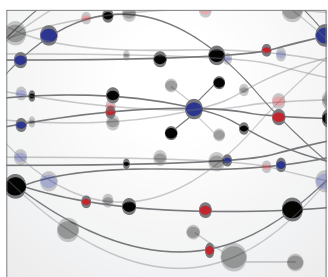

The Scientific World Journal
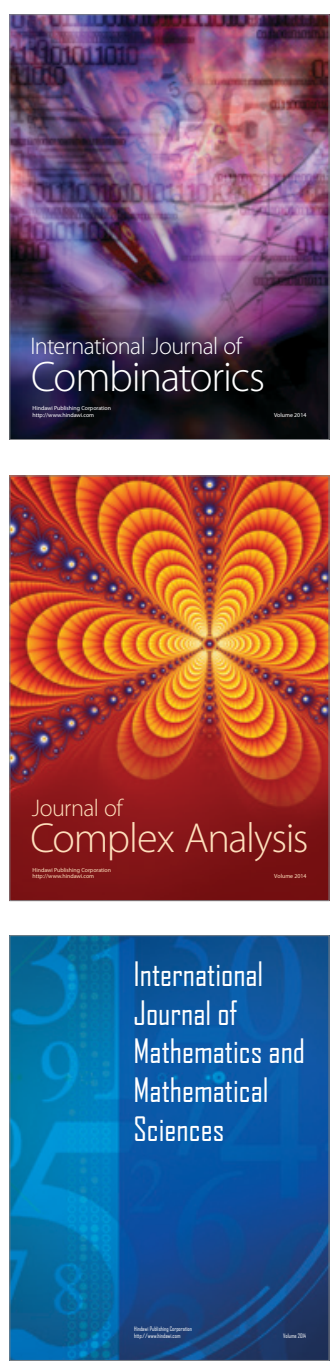
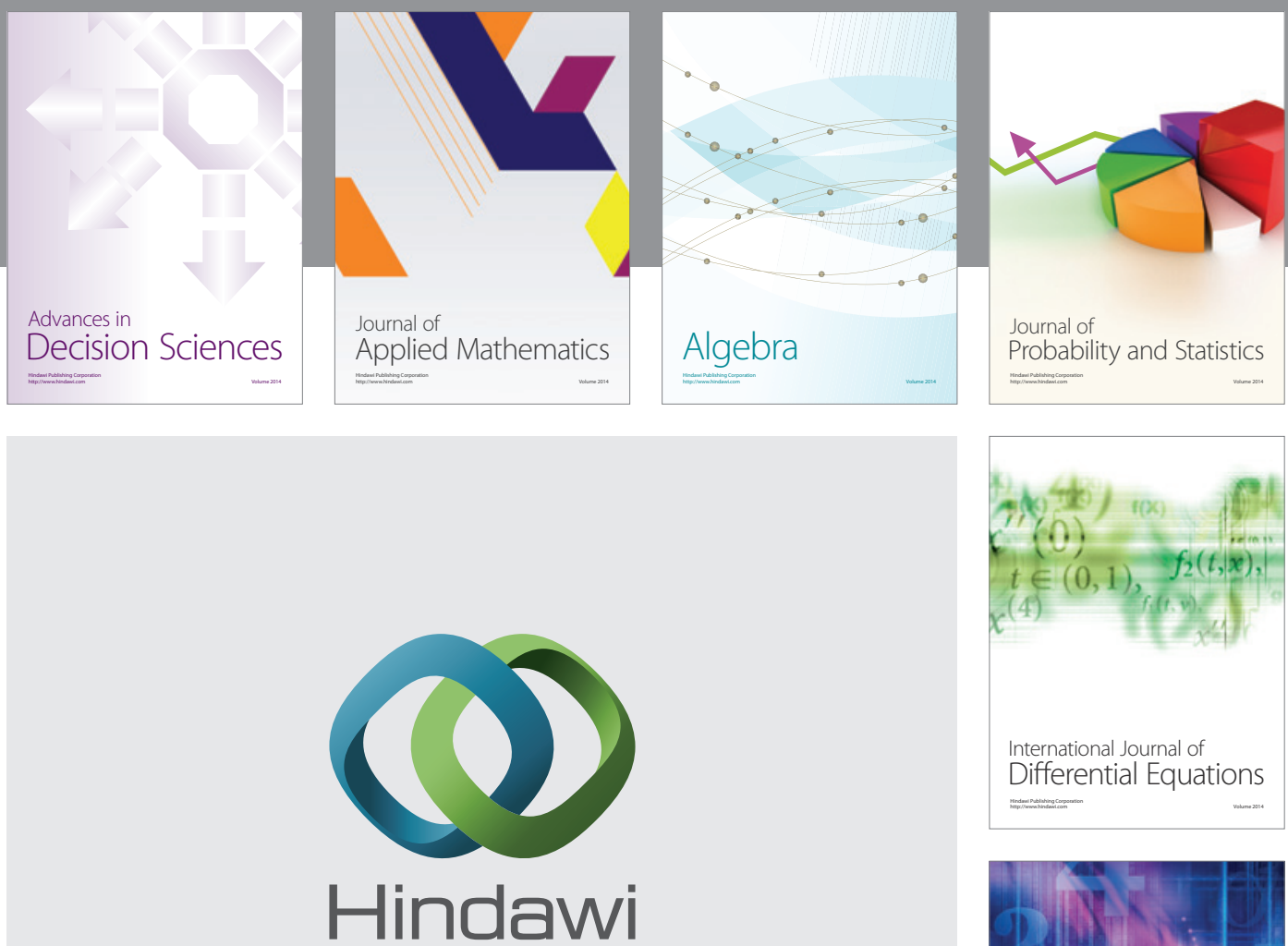

Submit your manuscripts at http://www.hindawi.com
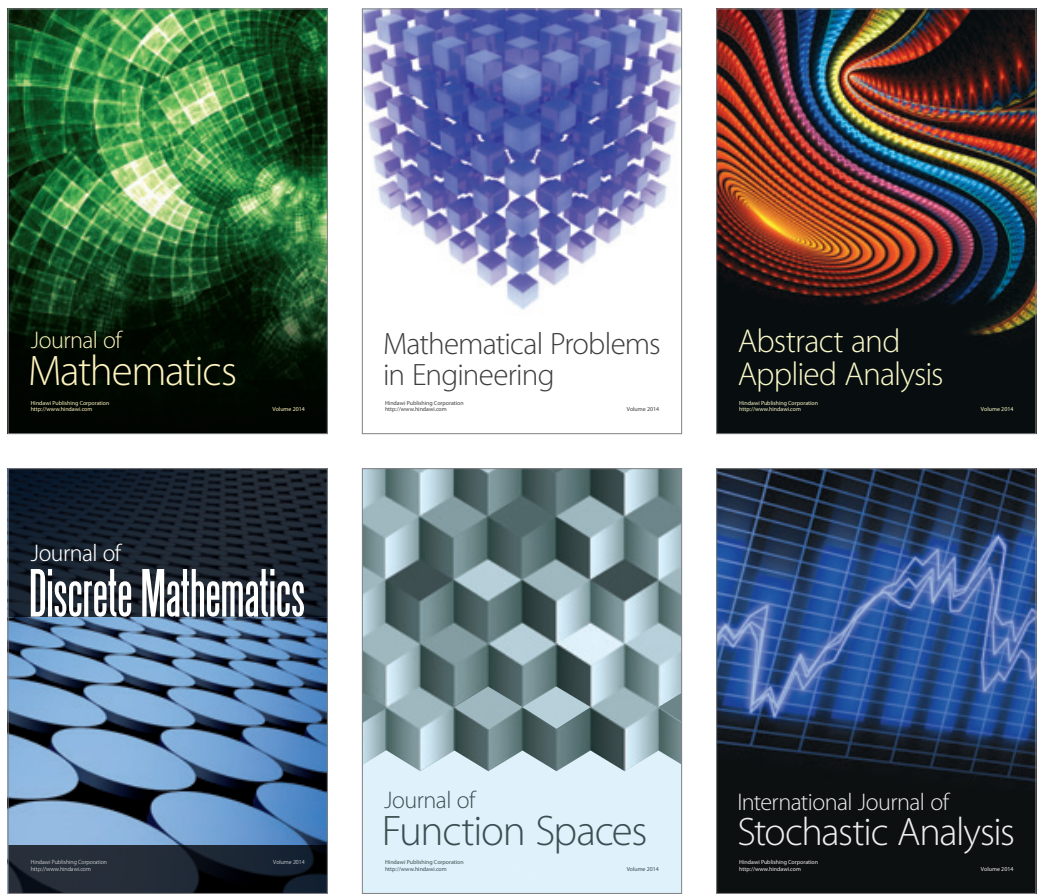

Journal of

Function Spaces

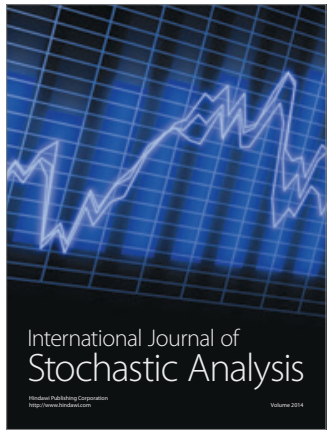

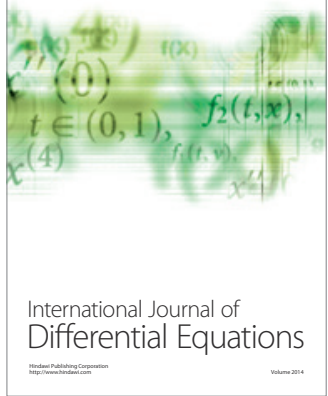
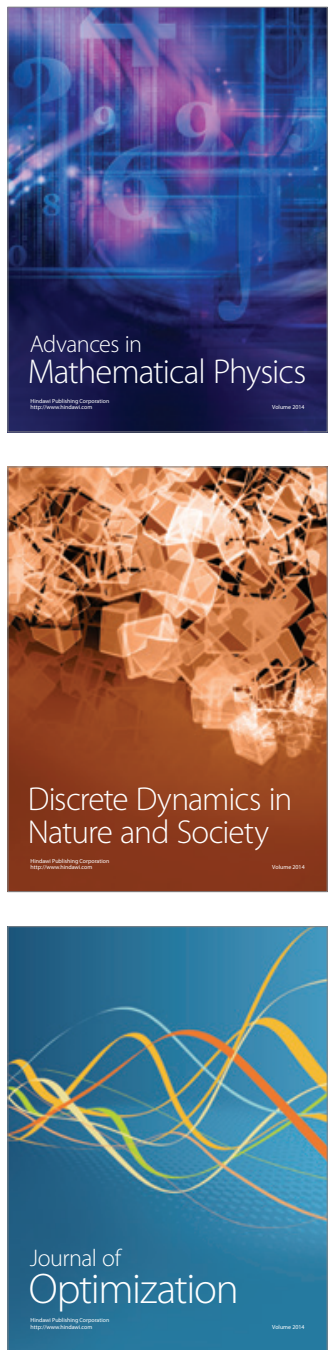